\title{
Gorenstein Toric Fano Varieties
}

\author{
BenJAMin NiLL \\ Mathematisches Institut, Universität Tübingen \\ Auf der Morgenstelle 10, 72076 Tübingen, Germany \\ e-mail: nill@everest.mathematik.uni-tuebingen.de
}

\begin{abstract}
We investigate Gorenstein toric Fano varieties by combinatorial methods using the notion of a reflexive polytope which appeared in connection to mirror symmetry. The paper contains generalisations of tools and previously known results for nonsingular toric Fano varieties. As applications we obtain new classification results, bounds of invariants and formulate conjectures concerning combinatorial and geometrical properties of reflexive polytopes.
\end{abstract}

\section{Introduction}

Gorenstein toric Fano Varieties, i.e., normal toric varieties whose anticanonical divisor is an ample Cartier divisor, correspond to reflexive polytopes introduced by Batyrev in Bat94. Reflexive polytopes are lattice polytopes containing the origin in their interior such that the dual polytope is also a lattice polytope. In Bat94 it was shown that the associated varieties are ambient spaces for CalabiYau hypersurfaces and together with their duals naturally yield candidates for mirror symmetry pairs. This has raised interest in this special class of lattice polytopes among physicists.

It is known that in fixed dimension $d$ there is only a finite number of isomorphism classes of $d$-dimensional reflexive polytopes. Using a computer program Kreuzer and Skarke classified all $d$-dimensional reflexive polytopes for $d \leq 4$ KS98, KS00a KS02. They found 16 isomorphism classes of $d$-dimensional reflexive polytopes for $d=2,4319$ for $d=3$, and 473800776 for $d=4$. This vast work gives mathematicians many examples of reflexive polytopes to test conjectures on.

There are many papers devoted to the investigation and classification of nonsingular toric Fano varieties WW82, Bat82, Bat99, Sat00, Deb01, Cas03a Cas03b. In this article we present new classification results, bounds of invariants and conjectures concerning Gorenstein toric Fano varieties by investigating combinatorial and geometrical properties of reflexive polytopes. Thereby we get not only results in higher dimensions but also explanations for interesting phenomena observed in lower dimensions.

In the forthcoming paper Nil04 the results achieved will be applied to investigate criteria for the group of automorphisms of a Gorenstein toric Fano variety to be reductive. These include the answer to a question related to the 
existence of an Einstein-Kähler metric. Furthermore a sharp bound on the dimension of the connected component of the reductive group of automorphisms will be proven.

The structure of this paper is as follows:

In section 1 we establish our notations and repeat the basic definitions.

In sections 2 and 3 two elementary technical tools are investigated and generalised that were previously already successfully used to investigate and classify nonsingular toric Fano varieties Bat99, Sat00, Cas03b.

In section 2 we investigate the properties of the projection of a reflexive polytope along a lattice point on the boundary. Thereby we can relate properties of a Gorenstein toric Fano variety to that of a lower-dimensional toric Fano variety (e.g., Cor. 2.5). As an application we give a new, purely combinatorial proof (Cor. 2.8) of a result due to Batyrev Bat99, Prop. 2.4.4] saying that the anticanonical class of a torus-invariant prime divisor of a nonsingular toric Fano variety is always numerically effective.

In section 3 we consider pairs of lattice points on the boundary of a reflexive polytope and show that in this case there exists a generalisation of the notion of a primitive relation as introduced by Batyrev in Bat91. As an application we show that there are constraints on the combinatorics of a reflexive polytope, in particular on the diameter of the edge-graph of a simplicial reflexive polytope (Cor. 3.2). Thereby we can prove that certain combinatorial types of polytopes cannot be realized as reflexive polytopes (Cor. 3.3. Cor. 3.4).

In sections 4 and 5 we present applications of the results of sections 2 and 3 .

In section 4 we give a short review of classification results of reflexive polytopes in low dimensions. We give a concise proof of the classification of reflexive polygons (Prop. 4.1), and a scetch of the proof of the classification of threedimensional Gorenstein toric Fano varieties with terminal singularities (Thm. 4.2).

In section 5 we formulate two conjectures (Conj. 5.2 Conj. [5.6) on the maximal number of vertices of a reflexive polytope, respectively a simplicial reflexive polytope. The currently best upper bound on the number of vertices of a smooth Fano polytope is due to Debarre Deb01, Thm. 8]. Here we give a generalisation to the case of a reflexive polytope (Thm. [5.4). The main result of the section is the verification of the conjecture on the maximal number of vertices of a simplicial reflexive polytope under the assumption of an additional symmetry of the polytope (Thm. 5.8). Furthermore we show that in fixed dimension a centrally symmetric simplicial reflexive polytope with the maximal number of vertices is even uniquely determined (Thm. [5.9). For the proofs we generalise a result due to Casagrande Cas03a, Thm. 2.4] to the case of a $\mathbb{Q}$-factorial Gorenstein toric Fano variety (Cor. [5.13) saying that the Picard number of a nonsingular toric Fano variety exceeds the Picard number of a torus-invariant prime divisor at most by three.

In section 6 we give sharp bounds on the number of lattice points in terminal Fano polytopes (Cor. 6.3) and centrally symmetric reflexive polytopes (Thm. 6.4).

Acknowledgement. The author would like to thank his thesis advisor Professor Victor Batyrev for posing problems, his advice and encouragement, as well as Professor Günter Ewald for giving reference to Wir97 and Professor Klaus Altmann for the possibility of giving a talk at the FU Berlin. The author 
would also like to thank Professor Maximillian Kreuzer for the support with the computer package PALP, the classification data and many examples.

The author was supported by DFG, Forschungsschwerpunkt "Globale Methoden in der komplexen Geometrie". This work is part of the author's thesis.

\section{Notation and basic definitions}

In this section we fix the notation for toric varieties and lay out the basic notions of toric Fano varieties. We refer to Ewa96 for combinatorial convexity and Ful93 Oda88 for toric varieties. In Bat94 reflexive polytopes were introduced. For a survey of (toric) Fano varieties see Deb01.

Let $N \cong \mathbb{Z}^{d}$ be a $d$-dimensional lattice and $M=\operatorname{Hom}_{\mathbb{Z}}(N, \mathbb{Z}) \cong \mathbb{Z}^{d}$ the dual lattice with $\langle\cdot, \cdot\rangle$ the nondegenerate symmetric pairing. As usual, $N_{\mathbb{Q}}=$ $N \otimes_{\mathbb{Z}} \mathbb{Q} \cong \mathbb{Q}^{d}$ and $M_{\mathbb{Q}}=M \otimes_{\mathbb{Z}} \mathbb{Q} \cong \mathbb{Q}^{d}$ (respectively $N_{\mathbb{R}}$ and $M_{\mathbb{R}}$ ) will denote the rational (respectively real) scalar extensions.

Throughout the paper the roles of $N$ and $M$ are interchangeable.

For a subset $S$ in a real vector space let $\operatorname{lin}(S)$ (respectively aff $(S), \operatorname{conv}(S)$, $\operatorname{pos}(S)$ ) be the linear (respectively affine, convex, positive) hull of $S$. A subset $P \subseteq M_{\mathbb{R}}$ is called a polytope, if it is the convex hull of finitely many points in $M_{\mathbb{R}}$. The boundary of $P$ is denoted by $\partial P$, the relative interior of $P$ by relint $P$. When $P$ is full-dimensional, its relative interior is also denoted by $\operatorname{int} P$. A face $F$ of $P$ is denoted by $F \leq P$, the vertices of $P$ form the set $\mathcal{V}(P)$, the facets of $P$ the set $\mathcal{F}(P) . P$ is called a lattice polytope, respectively rational polytope, if $\mathcal{V}(P) \subseteq M$, respectively $\mathcal{V}(P) \subseteq M_{\mathbb{Q}}$. An isomorphism of lattice polytopes is an isomorphism of the lattices such that the induced real linear isomorphism maps the polytopes onto each other.

We usually denote by $\triangle$ a complete fan in $M_{\mathbb{R}}$. The $k$-dimensional cones of $\triangle$ form a set $\triangle(k)$. The elements in $\triangle(1)$ are called rays, and given $\tau \in \triangle(1)$, we let $v_{\tau}$ denote the unique generator of $M \cap \tau$.

Since the polytope $P$ is throughout contained in $M_{\mathbb{R}}$, fans will also have cones mostly in $M_{\mathbb{R}}$ in contrast to the usual convention.

There are two possibilities to define a complete fan from a polytope $P \subseteq M_{\mathbb{R}}$ that is rational and $d$-dimensional:

First we can define the normal fan $\mathcal{N}_{P}$ of $P$, i.e., an element of $\mathcal{N}_{P}$ is the closed cone of inner normals of a face of $P$.

Second if the origin is contained in the interior of $P$, i.e., $0 \in \operatorname{int} P$, then we can define $\Sigma_{P}:=\{\operatorname{pos}(F): F \leq P\}$ as the fan that is spanned by $P$. In this case there is the important notion of the dual polytope

$$
P^{*}:=\left\{y \in N_{\mathbb{R}}:\langle x, y\rangle \geq-1 \forall x \in P\right\},
$$

that is a rational $d$-dimensional polytope with $0 \in \operatorname{int} P^{*}$. We have $\Sigma_{P^{*}}=\mathcal{N}_{P}$.

Duality means $\left(P^{*}\right)^{*}=P$. There is a natural combinatorial correspondence between $i$-dimensional faces of $P$ and $(d-1-i)$-dimensional faces of $P^{*}$ that reverses inclusion. For a facet $F \leq P$ we let $\eta_{F} \in N_{\mathbb{Q}}$ denote the uniquely determined inner normal satisfying $\left\langle\eta_{F}, F\right\rangle=-1$, so $\mathcal{V}\left(P^{*}\right)=\left\{\eta_{F}: F \in\right.$ $\mathcal{F}(P)\}$.

The dual of the product of $d_{i}$-dimensional polytopes $P_{i} \subseteq \mathbb{R}^{d_{i}}$ with $0 \in \operatorname{int} P_{i}$ for $i=1,2$ is given by

$$
\left(P_{1} \times P_{2}\right)^{*}=\operatorname{conv}\left(P_{1}^{*} \times\{0\},\{0\} \times P_{2}^{*}\right) \subseteq \mathbb{R}^{d_{1}} \times \mathbb{R}^{d_{2}} .
$$


Definition 1.1. Let $X$ be a normal complex variety and $K_{X}$ the canonical divisor of $X$, i.e., a Weil divisor of $X$ whose restriction to the regular locus defines the canonical sheaf there.

$K_{X}$ is $\mathbb{Q}$-Cartier, if there exists a positive integer $j$ such that $j K_{X}$ is a Cartier divisor. The smallest such $j$ is called the Gorenstein index $j_{X}$ of $X$.

A complex variety $X$ is called Fano variety (respectively weak Fano variety), if $X$ is projective, normal and the anticanonical divisor $-K_{X}$ is an ample (respectively nef and big) $\mathbb{Q}$-Cartier divisor.

A complex variety $X$ is called Gorenstein, iff $j_{X}=1$, i.e., $K_{X}$ is a Cartier divisor.

Back to the toric case: Let $\triangle$ be a complete fan in $M_{\mathbb{R}}$, we denote by $X:=X(M, \triangle)$ the associated complete normal toric variety.

$X$ is projective if and only if there is a $d$-dimensional lattice polytope $P \subseteq$ $M_{\mathbb{R}}$ with $0 \in \operatorname{int} P$ and $\triangle=\Sigma_{P}$, or equivalently, if there exists a $d$-dimensional lattice polytope $Q \subseteq N_{\mathbb{R}}$ with $\triangle=\mathcal{N}_{Q}$.

Set $G_{\triangle}:=\left\{v_{\tau}: \tau \in \triangle(1)\right\}$ and $G_{\sigma}:=G_{\triangle} \cap \sigma$ for $\sigma \in \triangle$.

It is well-known that $K_{X}:=-\sum_{\tau \in \triangle(1)} \mathcal{V}_{\tau}$ is a canonical divisor of $X$, where $\mathcal{V}_{\tau}$ denotes the torus-invariant prime divisor associated to the ray $\tau$. Furthermore $K_{X}$ is $\mathbb{Q}$-Cartier if and only if for all $\sigma \in \triangle$ the set $G_{\triangle} \cap \sigma$ is contained in an affine hyperplane.

Now we define the lattice polytope $P_{\triangle}:=\operatorname{conv}\left(G_{\triangle}\right) \subseteq M_{\mathbb{R}}$ with $0 \in \operatorname{int} P_{\triangle}$, and the rational dual polytope $Q_{\triangle}:=P_{\triangle}^{*} \subseteq N_{\mathbb{R}}$. Then $X$ is a toric Fano variety if and only if $\triangle=\Sigma_{P_{\Delta}}$, or equivalently, $\triangle=\mathcal{N}_{Q_{\Delta}}$. The Gorenstein index $j_{X}$ is the minimal $k$ such that $k Q_{\triangle}$ is a lattice polytope.

Whenever $K_{X}$ is $\mathbb{Q}$-Cartier we can explicitly compute the discrepancy of $X$ (see Deb01, Prop. 12]):

$$
\operatorname{discr}(X)=-1+\min \left\{\left\langle u_{\sigma}, v\right\rangle: \sigma \in \triangle(d), 0 \neq v \in \sigma \cap\left(M \backslash G_{\sigma}\right)\right\},
$$

thus a rational number in ]-1,1]. Especially $X$ has log-terminal singularities, i.e., $\operatorname{discr}(X)>-1$.

The following definitions are now convenient:

Definition 1.2. Let $P \subseteq M_{\mathbb{R}}$ be a $d$-dimensional lattice polytope with $0 \in \operatorname{int} P$.

- $P$ is called a Fano polytope, if the vertices are primitive lattice points.

- $P$ is called a canonical Fano polytope, if $\operatorname{int} P \cap M=\{0\}$.

- $P$ is called a terminal Fano polytope, if $P \cap M=\{0\} \cup \mathcal{V}(P)$.

- $P$ is called a smooth Fano polytope, if the vertices of any facet of $P$ form a $\mathbb{Z}$-basis of the lattice $M$.

Remark 1.3. Beware: In most papers (see Bat99 Sat00) a Fano polytope is assumed to be already a smooth Fano polytope. This more systematic notation was partly introduced in Deb01.

We have the following correspondence theorem:

Proposition 1.4. There is a correspondence between isomorphism classes of Fano polytopes and isomorphism classes of toric Fano varieties. 
Thereby canonical Fano polytopes correspond to toric Fano varieties with canonical singularities, i.e., $\operatorname{discr} X \geq 0$;

terminal Fano polytopes correspond to toric Fano varieties with terminal singularities, i.e., $\operatorname{discr} X>0$;

smooth Fano polytopes correspond to nonsingular toric Fano varieties.

There is the following important finiteness theorem (see Bor00 for a survey):

Theorem 1.5. For $\epsilon>0$ there exist only finitely many isomorphism classes of $d$-dimensional toric Fano varieties with discrepancy greater than $-1+\epsilon$.

For the weak case we define:

Definition 1.6. Let $P \subseteq M_{\mathbb{R}}$ be a Fano polytope spanning $\triangle$. Then a fan $\triangle^{\prime}$ is called a crepant refinement of $\triangle$, if $\triangle^{\prime}$ is a refinement of $\triangle$ in the usual sense and additionally for any $\tau^{\prime} \in \triangle^{\prime}(1)$ there exists a $\sigma \in \triangle$ such that $v_{\tau^{\prime}} \subseteq \operatorname{conv}\left(G_{\sigma}\right)$. When the toric variety associated to the fan $\triangle^{\prime}$ is again projective, the fan $\triangle^{\prime}$ is called a coherent crepant refinement.

Using the ramification formula (see [Deb01]) we see that such crepant refinements correspond to equivariant proper birational morphisms $f: X^{\prime}=X\left(M, \triangle^{\prime}\right) \rightarrow X=X(M, \triangle)$ with $K_{X^{\prime}}=f^{*} K_{X}$.

Proposition 1.7. Toric weak Fano varieties correspond uniquely up to isomorphism to coherent crepant refinements of fans spanned by Fano polytopes.

Finally let's recall the following definition:

Definition 1.8. A complex normal variety is called $\mathbb{Q}$-factorial, if any Weil divisor is $\mathbb{Q}$-Cartier.

For the next result (see Bat94, Thm. 2.2.24]) recall that a polytope is simplicial, if any facet is a simplex:

Proposition 1.9. $\mathbb{Q}$-factorial toric Fano varieties correspond uniquely up to isomorphism to simplicial Fano polytopes.

There exists a coherent crepant refinement by stellar subdivisions that resolves a weak toric Fano variety $X$ with canonical singularities to a $\mathbb{Q}$-factorial weak toric Fano variety $X^{\prime}$ with terminal singularities.

Such a morphism $X^{\prime} \rightarrow X$ is called a MPCP-desingularization in Bat94. $X$ is said to admit a coherent crepant resolution, if such an $X^{\prime}$ can be chosen to be nonsingular.

Reflexive polytopes naturally enter the picture when considering Gorenstein toric Fano varieties.

Definition 1.10. A Fano polytope whose dual is a lattice polytope is called reflexive polytope.

The following proposition is now straightforward.

Proposition 1.11. Gorenstein toric Fano varieties correspond uniquely up to isomorphism to reflexive polytopes.

Gorenstein toric weak Fano varieties correspond uniquely up to isomorphism to coherent crepant refinements of fans spanned by reflexive polytopes.

There is a complete duality of reflexive polytopes and their duals. 
Proposition 1.12. Let $P \subseteq M_{\mathbb{R}}$ be a d-dimensional lattice polytope with $0 \in$ $\operatorname{int} P$. Then the following conditions are equivalent:

1. $P$ is a reflexive polytope

2. $P$ is a lattice polytope and $P^{*}$ is a lattice polytope

3. $P^{*}$ is a reflexive polytope

If this holds, then $\operatorname{int} P \cap M=\{0\}$, i.e., $P$ is a canonical Fano polytope.

The proof is straightforward by using the following local property that characterises reflexive polytopes:

Lemma 1.13. Let $P \subseteq M_{\mathbb{R}}$ be a reflexive polytope.

For any $F \in \mathcal{F}(P)$ and $m \in F \cap M$ there is a $\mathbb{Z}$-basis $e_{1}, \ldots, e_{d-1}, e_{d}:=m$ of $M$ such that $F \subseteq\left\{x \in M_{\mathbb{R}}: x_{d}=1\right\}$; thus $\eta_{F}=-e_{d}^{*}$ in the dual $\mathbb{Z}$-basis $e_{1}^{*}, \ldots, e_{d}^{*}$ of $N$.

Proof. Since $\eta_{F} \in N$, the following short exact sequence splits

$$
0 \rightarrow\left\{x \in M:\left\langle\eta_{F}, x\right\rangle=0\right\} \rightarrow M \stackrel{\left\langle\eta_{F}, \cdot\right\rangle}{\rightarrow} \mathbb{Z} \rightarrow 0 .
$$

As an immediate corollary from 1.5 we get:

Corollary 1.14. Gorenstein toric (weak) Fano varieties have canonical singularities. In fixed dimension d there is only a finite number of isomorphism classes of d-dimensional Gorenstein toric Fano varieties.

Finally refering to 1.9 there is following result (see Bat94 and 2.6):

Proposition 1.15. For $d \leq 3$ any d-dimensional Gorenstein toric Fano variety admits a coherent crepant resolution.

For the proof we recall a convenient definition:

Definition 1.16. A lattice polytope $P \subseteq M_{\mathbb{R}}$ is called empty, if $P \cap M=\mathcal{V}(P)$.

Now 1.15 follows from [1.9] and the next well-known lemma:

Lemma 1.17. Let $P$ be a d-dimensional lattice polytope with $0 \in \operatorname{int} P$.

1. Let $d=2$. Lattice points $x, y$ form $a \mathbb{Z}$-basis if and only if $\operatorname{conv}(0, x, y)$ is an empty two-dimensional polytope. If $P$ is a canonical Fano polytope, this is fulfilled, if $x, y$ are lattice points on the boundary that are not contained in a common facet of $P$ and $x+y \neq 0$.

In particular a two-dimensional terminal Fano polytope is a smooth Fano polytope, and a two-dimensional canonical Fano polytope is a reflexive polytope.

2. Let $d=3$ and $P$ be reflexive. Three lattice points $x, y, z$ in a common facet of $P$ form a $\mathbb{Z}$-basis if and only if $\operatorname{conv}(x, y, z)$ is empty.

In particular a three-dimensional simplicial terminal reflexive polytope is a smooth Fano polytope. 


\section{Projecting along lattice points on the bound- ary}

In this section $P$ is a d-dimensional reflexive polytope in $M_{\mathbb{R}}$.

The projection map along a vertex of $P$ is an essential tool in investigating toric Fano varieties, since one hopes to get some information from the corresponding lower-dimensional variety (see Bat99 Cas03a). In the case of a reflexive polytope it is also useful to consider projecting along lattice points on the boundary of $P$ that are not necessarily vertices.

The following definitions will be used throughout the paper:

Definition 2.1. Let $x, y \in \partial P$ with $x \neq y$.

- $[x, y]:=\operatorname{conv}(x, y),] x, y]:=[x, y] \backslash\{x\},] x, y[:=[x, y] \backslash\{x, y\}$.

- $x \sim y$, if $[x, y]$ is contained in a face of $P$, i.e., $x$ and $y$ are contained in a common facet of $P$.

- The star set of $x$ is the set

$$
\operatorname{st}(x):=\{y \in \partial P: x \sim y\}=\bigcup\{F \in \mathcal{F}(P): x \in F\} .
$$

- The link of $x$ is the set

$$
\partial x:=\partial \operatorname{st}(x)=\bigcup\{G \leq P: G \subseteq \operatorname{st}(x), x \notin G\} .
$$

- $y \vdash x$ ( $y$ is said to be away from $x$ ), if $y$ is not in the relative interior of st $(x)$. Hence

$$
\partial x=\{y \in \operatorname{st}(x): y \vdash x\} .
$$

$y$ is away from $x$ iff there exists a facet that contains $y$ but not $x$, e.g., if $y$ is a vertex or $x \nsim y$. There is also a local criterion:

$$
y \vdash x \Longleftrightarrow x+\lambda(y-x) \notin P \forall \lambda>1 \text {. }
$$

The next proposition shows the important properties of the projection of a reflexive polytope along some lattice point on the boundary.

Proposition 2.2. Let $P \subseteq M_{\mathbb{R}}$ be a reflexive polytope. Let $v \in \partial P \cap M$. Define the quotient lattice $M_{v}:=M / \mathbb{Z} v$ and the canonical projection map along $v$

$$
\Pi=\Pi_{v}: M_{\mathbb{R}} \rightarrow\left(M_{v}\right)_{\mathbb{R}}=M_{\mathbb{R}} / \mathbb{R} v .
$$

Then $P_{v}:=\Pi_{v}(P)$ is a lattice polytope in $\left(M_{v}\right)_{\mathbb{R}}$ with $\mathcal{V}\left(P_{v}\right) \subseteq \Pi(\mathcal{V}(P))$ containing the origin in the interior.

1. Let $U$ be the set of elements $x \in P$ such that $x+\lambda v \notin P$ for all $\lambda>0$. Then we have a canonical bijection

$$
U \rightarrow P_{v} .
$$

We denote the inverse map by $\rho$.

For $S:=\operatorname{st}(v)$ we have

$$
U=S
$$

and thus

$$
P_{v}=\operatorname{conv}(\Pi(\mathcal{V}(P) \cap \partial v)) .
$$


2. The projection map induces a bijection $S \cap M \rightarrow P_{v} \cap M$.

3. The projection map induces a bijection $\partial v \rightarrow \partial P_{v} . \partial P_{v}$ is covered by the projection of all $(d-2)$-dimensional faces $C$ such that $C \in \mathcal{F}(F)$ for some facet $F$ of $P$ with $v \in F$ and $v \notin C ; \Pi(C)$ is contained in a facet of $P_{v}$.

4.

$$
\rho\left(\mathcal{V}\left(P_{v}\right)\right) \subseteq \mathcal{V}(P) \cap \partial v
$$

Let $z \in \mathcal{V}(P) \cap \partial v$. Then $\Pi(z) \in \partial P_{v}$. If $] v, z[$ is contained in the relative interior of a facet of $P$, then $\Pi(z) \in \mathcal{V}\left(P_{v}\right)$.

5. Let $F \in \mathcal{F}(P)$ with $v \in F$. Then

$$
\operatorname{pos}(\Pi(F)) \cap P_{v}=\Pi(F) .
$$

6. The image $\Pi(F)$ of a facet $F$ parallel to $v$, i.e., $\left\langle\eta_{F}, v\right\rangle=0$, is a facet of $P_{v}$. It is $\Pi^{-1}(\Pi(F)) \cap P=F$. There are at least $|\mathcal{V}(\Pi(F))|$-vertices of $F$ in $S$. Any point in $F \cap S$ is contained in a facet that contains $v$ and intersects $F$ in a $(d-2)$-dimensional face.

The preimage $\Gamma:=\Pi^{-1}\left(F^{\prime}\right) \cap P$ of a facet $F^{\prime}$ of $P_{v}$ is either a facet of $P$ parallel to $v$ or a $(d-2)$-dimensional face of $P$. In the last case $\Gamma \rightarrow F^{\prime}$ is an isomorphism, and there exists exactly one facet of $P$ that contains $\Gamma$ and $v$.

7. Suppose $-v \in P$. Then any facet of $P$ either contains $v$, or $-v$, or is parallel to $v$, i.e., a facet of the form $\Pi^{-1}\left(F^{\prime}\right) \cap P$ for $F^{\prime} \in \mathcal{F}\left(P_{v}\right)$.

8.

$$
\left(P_{v}\right)^{*} \cong P^{*} \cap v^{\perp} \text { as lattice polytopes. }
$$

$P_{v}$ is reflexive if and only if $P^{*} \cap v^{\perp}$ is a lattice polytope.

Proof. 1. Let $F$ be a facet of $P$ containing $v$ and $x$. If $\lambda>0$, then $\left\langle\eta_{F}, x+\lambda v\right\rangle=$ $-1-\lambda<-1$, so $x+\lambda v \notin P$. Hence $S \subseteq U$.

On the other hand let $x \in U$. Considering the polytope $P \cap \operatorname{lin}(v, x)$ we see there is a facet $F$ of $P$ not parallel to $v$ that contains $x$ with $\left\langle\eta_{F}, v\right\rangle<0$. Since $P$ is reflexive, we have $\left\langle\eta_{F}, v\right\rangle=-1$, hence $v \in F$ and $x \sim v$. This implies $S=U$.

2. Let $m^{\prime} \in P_{v} \cap M_{v}$. We have $u:=\rho\left(m^{\prime}\right) \in U=S$. So there exists a facet $F \in \mathcal{F}(P)$ with $v, u \in F$. By 1.13 there is a $\mathbb{Z}$-basis $e_{1}, \ldots, e_{d-1}, e_{d}=v$ of $M$ such that $\mathcal{V}(F) \subseteq\left\{x \in M_{\mathbb{R}}: x_{d}=1\right\}$, i.e., $\eta_{F}$ is the dual vector $-e_{d}^{*}$. Let $u=\lambda_{1} e_{1}+\ldots+\lambda_{d} e_{d}$ for $\lambda_{1}, \ldots, \lambda_{d} \in \mathbb{R}$. Now $e_{1}, \ldots, e_{d-1}$ is a $\mathbb{Z}$-basis of $M_{v}$. Therefore $\lambda_{1}, \ldots, \lambda_{d-1} \in \mathbb{Z}$. Since $u \in F$, we get $\lambda_{d}=u_{d}=\left\langle-\eta_{F}, u\right\rangle=1$, hence $u \in M$.

3. Let $z \in \partial v$. Then $z \vdash v$ and there exists a facet $F \in \mathcal{F}(P)$ with $v, z \in F$. Assume $z^{\prime}:=\Pi(z) \notin \partial P_{v}$. Then there is some $\lambda>1$ such that $\lambda z^{\prime} \in \partial P_{v}$. We have $x:=\rho\left(\lambda z^{\prime}\right) \sim v$. Since $x \in \operatorname{lin}(v, z)$ and $z \sim v$, this yields $x \in F$. This implies that $z$ is a proper convex combination of $v$ and $x$, a contradiction to $z \vdash v$. The remaining properties are as easily seen.

4. The first statements follow from the first and the third point. Now suppose $z \in \mathcal{V}(P) \cap S$ such that there is only one facet $F \in \mathcal{F}(P)$ that contains $v$ and 
$z$. Since $z \in \mathcal{V}(F)$ we can choose an affine hyperplane $H$ that intersects $F$ only in $z$ and is parallel to $v$. For $P_{v}:=\Pi_{v}(P)$ and $H^{\prime}:=\Pi_{v}(H)$ let $x^{\prime} \in H^{\prime} \cap P_{v}$. It remains to show that $x^{\prime}=z^{\prime}:=\Pi_{v}(z)$. So assume not. $H^{\prime}$ intersects $\Pi_{v}(F)$ only in $z^{\prime}$. Therefore $\rho\left(y^{\prime}\right) \notin F$ for all $\left.\left.y^{\prime} \in\right] z^{\prime}, x^{\prime}\right]$. Finiteness of $\mathcal{F}(P)$ implies that $z$ is contained in another facet $\neq F$ containing $v$, a contradiction.

5. This is proven as the third point.

6. The first statements follow from the third and the fourth point.

For the second statement let $\operatorname{dim}(\Gamma)=d-2$. Now observe that if $\Gamma \rightarrow$ $F^{\prime}$ were not injective, a facet containing $\Gamma$ necessarily would be parallel to $v$, so its image a facet containing $F^{\prime}$, a contradiction. Therefore $\Gamma \rightarrow F^{\prime}$ is an isomorphism of polytopes with respect to their affine hulls. Now choose $x \in \operatorname{relint} F^{\prime}$. Let $y:=\rho(x) \in S \cap \Gamma$. By assumption also $y \in \operatorname{relint} \Gamma$. Let $G \in \mathcal{F}(P)$ with $v, y \in G$. Then $\Gamma \subseteq G$, hence $G$ is one of the two facets containing $\Gamma$.

7. Let now $-v \in P$. Any facet $F \in \mathcal{F}(P)$ satiesfies $-1 \leq\left\langle\eta_{F}, v\right\rangle=$ $-\left\langle\eta_{F},-v\right\rangle \leq 1$. From this the statements follow.

8. Choose again a facet $F$ of $P$ with $v \in F$, and a $\mathbb{Z}$-basis $e_{1}, \ldots, e_{d}$ of $M$ such that $e_{d}=v$ and $e_{1}, \ldots, e_{d-1}$ is a $\mathbb{Z}$-basis of $M \cap \eta_{F}^{\perp} \cong M_{v}$. In these coordinates we get $\left(P_{v}\right)^{*}=\left\{y^{\prime} \in \mathbb{R}^{d-1}:\left\langle y^{\prime}, x^{\prime}\right\rangle \geq-1 \forall x^{\prime} \in P_{v}\right\}=\left\{y^{\prime} \in\right.$ $\left.\mathbb{R}^{d-1}:\left\langle\left(y_{1}^{\prime}, \ldots, y_{d-1}^{\prime}, 0\right),\left(x_{1}, \ldots, x_{d}\right)\right\rangle \geq-1 \forall x \in P\right\}=P^{*} \cap v^{\perp}$.

The last point and its proof are taken from [AKMS97, Thm. in Sect. 3].

Let's consider the algebraic-geometric interpretation of the projection map:

Let $\triangle:=\Sigma_{P}, X:=X\left(M, \Sigma_{P}\right)$. Let $v \in \mathcal{V}(P), \tau:=\operatorname{pos}(v) \in \triangle(1)$ and $\Pi=\Pi_{v}$. As in [0da88, 1.7] the fan $\triangle:=\{\Pi(\sigma): \sigma \in \triangle, \tau \leq \sigma\}=\{\operatorname{pos}(\Pi(F))$ : $F \in \mathcal{F}(P), v \in F\}$ defines the projective toric variety $\mathcal{V}_{\tau}$ that is the torus invariant prime divisor corresponding to the ray $\tau$.

On the other hand there is the projected polytope $P_{v}:=\Pi(P)$ that spans a fan $\triangle_{v}$ in $\left(M_{v}\right)_{\mathbb{R}}$, we denote the corresponding projective toric variety by $X_{v}$. In the following we discuss how and when $\mathcal{V}_{\tau}$ and $X_{v}$ are related.

We choose a triangulation $\mathcal{T}:=\left\{T_{k}\right\}$ of $\partial v$ into simplicial lattice polytopes. Then $\triangle_{\mathcal{T}}:=\left\{\operatorname{pos}\left(\Pi\left(T_{k}\right)\right)\right\}$ is a simplicial fan in $\left(M_{v}\right)_{\mathbb{R}}$ with corresponding $\mathbb{Q}$ factorial complete toric variety $X_{\mathcal{T}}$. From prop. [2.2 $(5)$ it follows that $\triangle_{\mathcal{T}}$ is a common refinement of $\bar{\triangle}$ and $\triangle_{v}$. Especially there are induced proper birational morphisms $X_{\mathcal{T}} \rightarrow \mathcal{V}_{\tau}$ and $X_{\mathcal{T}} \rightarrow X_{v}$.

In general $\triangle_{v}$ is not a refinement of $\bar{\triangle}$. However in the case that $P$ is simplicial, we can choose $\mathcal{T}$ obviously in such a way that $\triangle_{\mathcal{T}}=\bar{\triangle}$, in particular this implies that $\bar{\triangle}$ is a refinement of $\triangle_{v}$.

In order to draw conclusions about the canonical divisor and singularities of these lower-dimensional toric varieties there is the following sufficient assumption:

$$
\exists f \in \mathbb{N}_{>0}: \forall w \in \mathcal{V}(P) \cap \partial v:|[v, w] \cap M|-1=f .
$$

Suppose this condition holds. For any $w \in \mathcal{V}(P) \cap \partial v$ the second point in the proposition implies $\left|\left[0, \Pi_{v}(w)\right] \cap M_{v}\right|-1=f$. Furthermore let $w^{\prime} \in \mathcal{V}\left(P_{v}\right)$, $\tau^{\prime}:=\operatorname{pos}\left(w^{\prime}\right)$. Since prop. [2.2(4) implies $\rho\left(w^{\prime}\right) \in \mathcal{V}(P) \cap \partial v$, the previous consideration yields $v_{\tau^{\prime}}=(1 / f) w^{\prime}$, hence $P_{\triangle_{v}}=(1 / f) P_{v}$ (as defined in section 11). Therefore $-K_{X_{v}}$ is an ample $\mathbb{Q}$-Cartier divisor, i.e., $X_{v}$ is a toric Fano variety. 
Definition 2.3. $X$, respectively $P$, is called semi-terminal, if for all $v \in \mathcal{V}(P)$ condition (2) holds for $f=1$, i.e., $[v, w] \cap M=\{v, w\}$ for all $w \in \mathcal{V}(P) \cap \partial v$.

Proposition 2.4. Let $P \subseteq M_{\mathbb{R}}$ be a reflexive polytope.

1. $P$ is semi-terminal iff $P_{v}$ is a Fano polytope for all $v \in \mathcal{V}(P)$.

2. $P$ is terminal iff $P_{v}$ is a canonical Fano polytope for all $v \in \mathcal{V}(P)$.

Proof. 1. From left to right: This holds since $P_{\triangle_{v}}=P_{v}$ is a Fano polytope.

From right to left: Let $v \neq w \in \mathcal{V}(P)$ with $v \sim w$. Choose $C$ and $F$ as in prop. 2.2(3) such that $w \in \mathcal{V}(C)$. By prop. 2.2(3) we see that $F^{\prime}:=$ aff $\left(\Pi_{v}(C)\right) \cap P_{v}$ is a facet of $P_{v}$. Hence by prop. [2.2(6) for $\Gamma:=\Pi_{v}^{-1}\left(F^{\prime}\right) \cap P$ we have either $\Gamma=G$ for a facet $G \in \mathcal{F}(P)$ that is parallel to $v$ or $\Gamma=C$. In the first case $\left\langle\eta_{G}, v\right\rangle=0$ and $\left\langle\eta_{G}, w\right\rangle=-1$, so $|[v, w] \cap M|=2$. In the second case obviously $F^{\prime}=\Pi_{v}(C)$, so $\Pi_{v}(w) \in \mathcal{V}\left(F^{\prime}\right)$, hence by assumption a primitive lattice point. From prop. $[2.2(2)$ we get $|[v, w] \cap M|=2$.

2. From left to right: Let $v \in \mathcal{V}(P), 0 \neq m^{\prime} \in M_{v} \cap P_{v}$. In the notation of prop. [2.2(1,2) we get $\rho\left(m^{\prime}\right) \in M \cap \partial P \subseteq \mathcal{V}(P)$ by assumption. Hence $m^{\prime}=\Pi_{v}\left(\rho\left(m^{\prime}\right)\right) \in \partial P_{v}$ by prop. [2.2(4).

From right to left: Assume there is a $w \in \partial P \cap M, w \notin \mathcal{V}(P)$. Then $w$ is a proper convex combination of vertices of $P$ contained in a common facet. Let $v$ be one of them. Then $\Pi_{v}(w)$ is obviously in the interior of $P_{v}$, a contradiction.

Corollary 2.5. Let $P \subseteq M_{\mathbb{R}}$ be a reflexive polytope.

Then the following two statements are equivalent:

\section{X has terminal singularities}

2. $X$ is semi-terminal and $X_{v}$ has canonical singularities for any $v \in \mathcal{V}(P)$

If this holds, then $X_{v}$ is a toric Fano variety for any $v \in \mathcal{V}(P)$.

In particular we see that terminality is a necessary condition for obtaining a reflexive polytope under projection, however not sufficient for $d \geq 4$.

Example 2.6. Let $d=4$, and $e_{1}, \ldots, e_{4}$ a $\mathbb{Z}$-basis of $M$. We define the simplicial centrally symmetric reflexive polytope $P:=\operatorname{conv}\left( \pm\left(2 e_{1}+e_{2}+e_{3}+\right.\right.$ $\left.\left.e_{4}\right), \pm e_{2}, \pm e_{3}, \pm e_{4}\right)$. Then $P$ is combinatorially a crosspolytope, has 8 vertices and 16 facets. It is a terminal Fano polytope but not a smooth Fano polytope, so especially it admits no crepant resolution. The projection $P_{e_{4}}$ along the vertex $e_{4}$ has 6 vertices, $P_{e_{4}}$ is even a terminal Fano polytope but not reflexive. This polytope is taken from Wir97 where it is used in a different context.

Now let's look at $\mathcal{V}_{\tau}$ : To ensure that the canonical divisor of $\mathcal{V}_{\tau}$ is $\mathbb{Q}$-Cartier, we need in general the $\mathbb{Q}$-factoriality of $X$. So let $P$ be simplicial and assume again that condition (2) holds. Then $\bar{\triangle}$ is a coherent crepant refinement of $\triangle_{v}$. Hence $-K_{\mathcal{V}_{\tau}}$ is a nef $\mathbb{Q}$-Cartier divisor, i.e., $\mathcal{V}_{\tau}$ is a toric weak Fano variety. From cor. 2.5] we get: 
Corollary 2.7. Let $X$ be a $\mathbb{Q}$-factorial Gorenstein toric Fano variety.

The following two statements are equivalent:

1. X has terminal singularities

2. $X$ is semi-terminal and $\mathcal{V}_{\tau}$ has terminal singularities for any $\tau \in \triangle(1)$

If this holds, then $\mathcal{V}_{\tau}$ is a $\mathbb{Q}$-factorial toric weak Fano variety for any $\tau \in \triangle(1)$.

Finally to additionally derive the Gorenstein property, i.e., that the canonical divisor is $\mathbb{Z}$-Cartier, we need a stronger assumption, that is trivial in the case of a smooth Fano polytope:

For any $F \in \mathcal{F}(P)$ with $v \in F$ and $C \in \mathcal{F}(F)$ with $v \notin C$

there exist $w_{1}, \ldots, w_{d-1} \in C \cap M$ such that

$w_{1}-v, \ldots, w_{d-1}-v$ is a $\mathbb{Z}$-basis (of $\left.\eta_{F}^{\perp} \cap M\right)$.

If this condition is fulfilled, then (2) holds for $f=1, P_{v}$ is reflexive by prop. [2.2(3), and $X_{v}$ is a Gorenstein toric Fano variety. If $P$ is also simplicial, then $\mathcal{V}_{\tau}$ is a Gorenstein toric weak Fano variety.

Suppose now $X$ is semi-terminal, simplicial and $\mathcal{V}_{\tau}$ is nonsingular for any $\tau \in \triangle(1)$. It follows from 1.13 that (3) holds for any $v \in \mathcal{V}(P)$. Then $P_{v}$ is reflexive, in particular canonical for any $v \in \mathcal{V}(P)$, hence cor. 2.5] implies that $P$ is terminal. Since $P$ is also simplicial, the assumption implies that $P$ is already a smooth Fano polytope. We have proven the following corollary:

Corollary 2.8. Let $X$ be a $\mathbb{Q}$-factorial Gorenstein toric Fano variety.

The following two statements are equivalent:

1. $X$ is nonsingular

2. $X$ is semi-terminal and $\mathcal{V}_{\tau}$ is nonsingular for any $\tau \in \triangle(1)$

If this holds, then $\mathcal{V}_{\tau}$ is a $\mathbb{Q}$-factorial toric weak Fano variety for any $\tau \in$ $\triangle(1)$, and $X_{v}$ is a Gorenstein toric Fano variety admitting the coherent crepant resolution $\mathcal{V}_{\tau} \rightarrow X_{v}$ for any $v \in \mathcal{V}(P), \tau=\operatorname{pos}(v)$.

The important fact that the projection of a smooth Fano polytope is reflexive was already proven by Batyrev in [Bat99. Prop. 2.4.4], however he used the notion of a primitive relation Bat91 and results of Reid about the Mori cone Rei83.

There is now a generalisation of this result to the class of toric Fano varieties with locally complete intersections. These varieties were thoroughly investigated in DHZ01, where it was proven that they admit coherent crepant resolutions.

Proposition 2.9. Let $X$ be a Gorenstein toric Fano variety that has singularities that are locally complete intersections.

Then the following three statements are equivalent:

1. $X$ is semi-terminal

2. $X$ has terminal singularities

3. Any facet of $P$ can be embedded as a lattice polytope in $[0,1]^{d-1}$ 
If this holds, then $X_{v}$ is a Gorenstein toric Fano variety for any $v \in \mathcal{V}(P)$. If additionally $X$ is $\mathbb{Q}$-factorial, then $X$ is nonsingular.

Proof. The facets of the corresponding reflexive polytope $P$ are so called Nakajima polytopes, a comprehensive description can be found in DHZ01. Using their results it is straightforward to prove the following statement by induction on $n$ :

Let $v$ be a vertex of an $n$-dimensional Nakajima polytope $F$ in a lattice $M$ such that $\left|\left[w, w^{\prime}\right] \cap M\right|=2$ for all $w, w^{\prime} \in \mathcal{V}(P), w \neq w^{\prime}$. Then $F$ is empty, can be embedded as a lattice polytope in $[0,1]^{n}$, and for any facet $C \in \mathcal{F}(F)$ with $v \notin C$ there exist $n$ vertices $w_{1}, \ldots, w_{n}$ of $C$ such that $w_{1}-v, \ldots, w_{n}-v$ is a $\mathbb{Z}$-basis.

From this result the proposition is obvious using condition (3).

\section{Pairs of lattice points on the boundary}

Throughout the section let $P$ be a d-dimensional reflexive polytope in $M_{\mathbb{R}}$.

In Bat91 the important notions of primitive collections and primitive relations were defined for nonsingular projective toric varieties, and used in Bat99. for the classification of four-dimensional smooth Fano polytopes. Unfortunately these useful tools cannot simply be generalised to the class of reflexive polytopes. However the next proposition shows that in the simplest yet most important case of a primitive collection of order two, i.e., a pair of lattice points on the boundary that is not contained in a common face, we still have a kind of generalised primitive relation:

Proposition 3.1. Let $P \subseteq M_{\mathbb{R}}$ be a reflexive polytope, $v, w \in \partial P \cap M, v \neq w$.

Exactly one of the following three statements holds:

1. $v \sim w$

2. $v+w=0$

3. $v+w \in \partial P$

Let the third condition be fulfilled. Then it holds:

$v, w$ is a $\mathbb{Z}$-basis of $\operatorname{lin}(v, w) \cap M$. There exists exactly one pair $(a, b) \in \mathbb{N}_{>0}^{2}$ with $z:=z(v, w):=a v+b w \in \partial P$ such that $v \sim z$ and $w \sim z$. Moreover:

i. $a=1$ or $b=1$. $a=|[w, z] \cap M|-1$ and $b=|[v, z] \cap M|-1$. If $F \in \mathcal{F}(P)$ with $v, z \in F$, then $\left\langle\eta_{F}, w\right\rangle=\frac{a-1}{b}$.

ii. Any facet containing $z($ or $v+w)$ contains exactly one of the points $v$ or $w$.

iii. For any $F \in \mathcal{F}(P)$ containing $v$ and $z$ there exists a facet $G \in \mathcal{F}(P)$ containing $w$ and $z$ such that $F \cap G$ is a $(d-2)$-dimensional face of $P$.

iv. If $z \in \mathcal{V}(P), b=1$, and $] v, z[$ is contained in the relative interior of a facet of $P$, then $[w, z]$ is contained in an edge. 
Proof. Let $v \nsim w$ and $v+w \neq 0$. The first condition implies that for any facet $F \in \mathcal{F}(P)$ we have $\left\langle\eta_{F}, v+w\right\rangle=\left\langle\eta_{F}, v\right\rangle+\left\langle\eta_{F}, w\right\rangle>-2$. However reflexivity of $P$ implies that this must be a natural number greater or equal to -1 , so $v+w \in P$ by duality. We get $0 \neq v+w \in \partial P$, because $P$ is canonical.

$v, w$ is a $\mathbb{Z}$-basis by $1.17(1)$.

Let $F$ be a facet of $P$ containing $v+w$. We may assume $\left\langle\eta_{F}, v\right\rangle=-1$ and $\left\langle\eta_{F}, w\right\rangle=0$. This implies $v \sim v+w$. We can use this consideration again for the pair $v+w, w$. Since $F \cap M$ is finite, this eventually yields a natural number $b \in \mathbb{N}_{>0}$ such that $z=v+b w \in F$ and $w \sim z$. In particular $a=1$. This proves the existence of $z$ and i.

ii. Let $F^{\prime} \in \mathcal{F}(P)$ with $z \in F^{\prime}$. Assume $v, w \notin F^{\prime}$, hence $-1=\left\langle\eta_{F^{\prime}}, z\right\rangle=$ $a\left\langle\eta_{F^{\prime}}, v\right\rangle+b\left\langle\eta_{F^{\prime}}, w\right\rangle \geq 0$, a contradiction.

iii. Let $F \in \mathcal{F}(P)$ with $v, z \in F$. We set $w^{\prime}:=(a-1) v+w \in \partial P \cap M$, hence $z=z\left(v, w^{\prime}\right)$. Since $\left\langle\eta_{F}, w^{\prime}\right\rangle=0$ and $z \in F \cap \operatorname{st}\left(w^{\prime}\right)$, prop. [2.2(6) applied to $\Pi_{w^{\prime}}$ implies that there exists a facet $G \in \mathcal{F}(P)$ containing $z$ and $w^{\prime}$ that intersects $F$ in a $(d-2)$-dimensional face. Since obviously $v \notin G$, it follows from the second point that $w^{\prime} \in G$. This immediately yields $w \in G$.

iv. Follows from prop. 2.2(4) applied to $\Pi_{v}$.

The next figure illustrates the proposition:

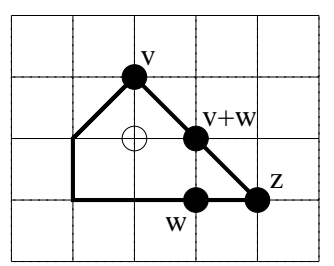

The symmetric relation $\sim$ defines a graph $\mathcal{W}(P)$ on $\partial P \cap M$. From the previous proposition we can now easily derive the following corollary about combinatorial properties of reflexive polytopes:

Corollary 3.2. Let $P \subseteq M_{\mathbb{R}}$ be a reflexive polytope.

1. Any pair of points in $\partial P \cap M$ can be connected by at most three edges of the graph $\mathcal{W}(P)$, with equality only possibly occuring for a centrally symmetric pair of points. In particular the diameter of the graph $\mathcal{W}(P)$ is at most three.

2. The previous statements also hold for the restriction of $\mathcal{W}(P)$ to the set of vertices, which is a purely combinatorial object. In the case of a simplicial polytope this is just the usual edge-graph on the vertices of $P$.

3. By dualizing we get that a pair of facets of a reflexive polytope is either parallel, contains a common vertex, or does have mutually non-trivial intersection with another facet.

Without using the existing classification of two-dimensional reflexive polytopes (see prop. 4.1) the proposition and the corollary yield an immediate application in the case of $d=2$ (for the proof of the second point use statement $\mathrm{i}$ of the proposition). 
Corollary 3.3. Let $P$ be a two-dimensional reflexive polytope.

1. $P$ has at most six vertices; equality occurs iff $P$ is of type 6 a in prop. 4.1 .

2. Any facet of $P$ contains at most five lattice points; there exists a facet with five lattice points iff $P$ is of type $8 \mathrm{c}$ in prop. 4.1 .

This first point is also a direct consequence of [PR00, Thm. 1] saying that $|\partial P \cap M|+\left|\partial P^{*} \cap N\right|=12$, where however no direct combinatorial proof is known that does not use some kind of induction.

Another application is to show that certain combinatorial isomorphismtypes of polytopes cannot be realized as reflexive polytopes. As an example have a look at the regular polyhedra (see for instance [Sti01]).

Corollary 3.4. In any dimension the combinatorial type of a d-simplex, a $d$ cube, and a d-crosspolytope can be realized as a reflexive polytope. There is no three-dimensional reflexive polytope that is combinatorially isomorphic to a dodecahedron or an icosahedron. There is no four-dimensional reflexive polytope that is combinatorially isomorphic to the 120-cell or 600-cell.

Proof. The first statement is trivial.

Let $P$ be a reflexive polytope and $d=3$. By duality we can assume that $P$ is combinatorially isomorphic to an icosahedron. Cor. [3.2(2) yields that $P$ is centrally symmetric. However any three-dimensional centrally symmetric simplicial reflexive polytope has at most 8 vertices as will be proven in thm. 5.9 .

Finally by cor. 3.2 and duality it is enough to note that the diameter of the edge-graph of the simplicial 600-cell is larger than three (see [Sti01, Fig. 5]).

It is now an astonishing observation (see KS00b) that the self-dual 24-cell can be uniquely realized as a reflexive polytope with vertices $\left\{ \pm e_{i}: i=1, \ldots, 4\right\}$ $\cup\left\{ \pm\left(e_{i}-e_{j}\right): i=1,2, j=2,3,4, j>i\right\} \cup\left\{ \pm\left(e_{i}-e_{3}-e_{4}\right): i=1,2\right\} \cup\left\{ \pm\left(e_{1}+\right.\right.$ $\left.\left.e_{2}-e_{3}-e_{4}\right)\right\}$ for $e_{1}, \ldots, e_{4}$ a $\mathbb{Z}$-basis of $M$. It is even centrally symmetric and terminal. Here it is interesting to note the necessity of these conditions:

Corollary 3.5. Let $P$ be a four-dimensional reflexive polytope $P$ that is combinatorially a 24-cell. Then $P$ has to be centrally symmetric and terminal.

Proof. Let $v$ be a vertex of $P$. Now choose the vertex $w \in \mathcal{V}(P)$ corresponding to the usual antipodal point. Assume $v+w \neq 0$. It is easy to see (see Sti01, Fig. 4]) that the intersection of a facet containing $v$ and a facet containing $w$ is empty or consists of a unique vertex $z$ where $] v, z[$ and $] w, z[$ are contained in the relative interiors of these facets. This implies $v \not w w$ and $z(v, w)=z \in \mathcal{V}(P)$, a contradiction to the last point of prop. 3.1

The terminality of $P$ can be proven in an analogous way. 


\section{Classification results in low dimensions}

Smooth Fano polytopes, as they form the most important class of reflexive polytopes, were intensively studied over the last decade by Batyrev Bat91 Bat99, Casagrande Cas03b, Cas03a], Debarre [Deb01, Sato [Sat00, et al. It could be rigorously proven that there are 18 smooth Fano polytopes for $d=3$ (see Bat82 WW82]) and 124 for $d=4$ (see Bat99, Sat00) up to isomorphism.

Here we will have a look at recent classification results of reflexive polytopes in low dimensions.

For $d=1$ the polytope $[-1,1]$ corresponding to $\mathbb{P}^{1}$ is the only Fano polytope. For $d=2$ any canonical Fano polytope is reflexive by $1.17(1)$, and these isomorphism classes can be easily classified (e.g., see KS97 or Sat00 Thm. $6.22]$ ). For the convenience of the reader and later reference we will give the list of the 16 isomorphism classes of reflexive polygons as well as a simple proof.

Proposition 4.1. There are exactly 16 isomorphism classes of two-dimensional reflexive polytopes (the number in the labels are the number of lattice points on the boundary):

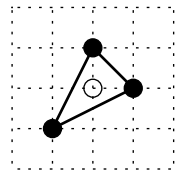

3

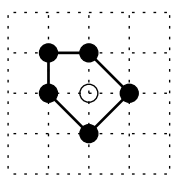

$5 a$

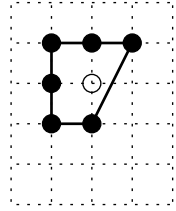

$6 c$

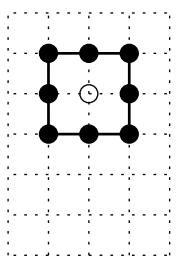

$8 a$

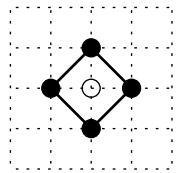

$4 a$

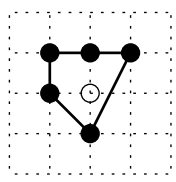

$5 b$

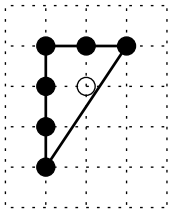

$6 d$

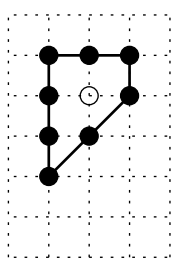

$8 b$

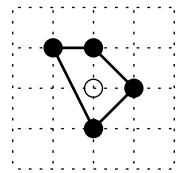

$4 \mathrm{~b}$

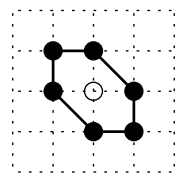

$6 a$

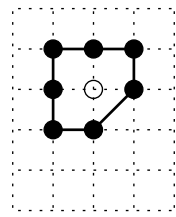

$7 a$

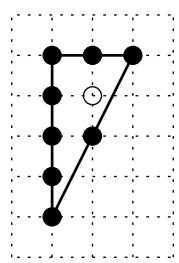

$8 c$

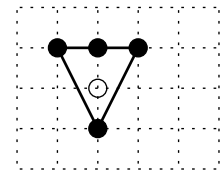

$4 \mathrm{c}$

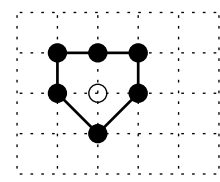

$6 b$

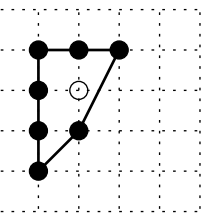

$7 \mathrm{~b}$

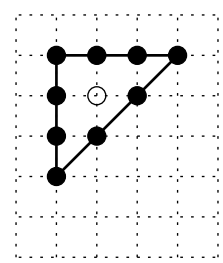

9 
Proof. Let $P$ be a two-dimensional reflexive polytope. We distinguish three different cases:

1. Any facet of $P$ contains only two lattice points, i.e., $P$ is a terminal Fano polytope. There are three different cases (see prop. 3.1):

(a) $P$ is combinatorially a triangle.

By 1.17(1) we may assume that $(1,0),(0,1)$ are vertices of $P$. Let $x$ be the third vertex. From prop. 2.4 $(2)$ it follows that the projection of $P$ along $(1,0)$ is a canonical Fano polytope, i.e., isomorphic to $[-1,1]$, hence $x_{2}=-1$. By projecting along $(0,1)$ we get $x_{1}=-1$, so $P$ is of type 3 .

(b) There exist three vertices $u, v, w \in \mathcal{V}(P)$ with $u+w=v$.

Since $P$ is a terminal Fano polytope, prop. 3.1 implies that $u \sim v$ and $w \sim v$, and we may assume $u=(-1,1), v=(0,1), w=(1,0)$. Again projecting along $v$ yields $P \cap\{(-1, x): x \in \mathbb{Z}\} \subseteq\{(-1,0),(-1,1)\}$, $P \cap\{(0, x): x \in \mathbb{Z}\} \subseteq\{(0,-1),(0,0),(0,1)\}, P \cap\{(1, x): x \in \mathbb{Z}\} \subseteq$ $\{(1,-1),(1,0)\}$. We get as possible types $4 b, 5 a, 6 a$.

(c) Any two vertices that are no neighbours are centrally symmetric.

This immediately implies that $P$ is of type $4 a$.

2. There exists a facet $F$ containing exactly one lattice point in relint $F$.

We may assume $\mathcal{V}(F)=\{(-1,1),(1,1)\}$. Then by prop. 2.2(1) we have $P \subseteq\left\{x \in \mathbb{R}^{2}:-1 \leq x_{1} \leq 1, x_{2} \leq 1\right\}$. Since $(0,-1)$ is not contained in $\operatorname{int} P$, we get $P \subseteq\left\{x \in \mathbb{R}^{2}: x_{2} \geq-3\right\}$. From this we readily derive the next ten isomorphism types $4 c, 5 b, 6 b, 6 c, 6 d, 7 a, 7 b, 8 a, 8 b, 8 c$.

3. The remaining case.

We may assume $\mathcal{V}(F)=\{(-1,1),(a, 1)\}$ for $a \in \mathbb{N}, a \geq 2$. Let $v \in \mathcal{V}(P)$ with $v_{2} \leq-1$ minimal. As $(1,0)$ is not in the interior of $P$, we have $v_{1} \leq 0$. Then by assumption necessarily $\operatorname{conv}((-1,1),(-1,-2),(2,1)) \subseteq P$. This must be an equality, hence $P$ is of type 9 .

The proof includes the statement that there are exactly five toric Del Pezzo surfaces, i.e., two-dimensional nonsingular toric Fano varieties, a result that can also be proven by birational factorisation Oda88, Prop. 2.21], primitive relations [Bat91] or determinants Ewa96 Thm. V.8.2].

In general even for $d=3$ there are too many reflexive polytopes to give a classification by pencil and paper. In Con02 reflexive simplices are classified using the notion of weights. Kreuzer and Skarke described in KS97, KS98 KS00a a general algorithm to classify reflexive polytopes in fixed dimension $d$. Using their computer program PALP (see KS02) they applied their method for $d \leq 4$, and found 4319 reflexive polytopes for $d=3$ and 473800776 for $d=4$. They also described how to find a normal form of lattice polytopes, toric fibrations and symmetries.

However it is still interesting to find rigorous mathematical proofs of observations and classification results of smaller classes of reflexive polytopes by directly using their intrinsic properties. There is the following result by the author: 
Theorem 4.2. There are 100 three-dimensional terminal reflexive polytopes. A three-dimensional reflexive polytope is terminal if and only if any facet is either a simplex where the vertices form a $\mathbb{Z}$-basis or a parallelogram $\operatorname{conv}(v, w, x, y)$ where $v, x, w$ form $a \mathbb{Z}$-basis and satisfy the equation $v+x=w+y$.

A complete proof is contained in the thesis of the author. It relies on the notion of an $A S$-point, i.e., a vertex that is both additive, i.e., the sum of two other vertices, and symmetric, i.e., its antipodal point is also a vertex. If no such AS-point exists, we use prop. 2.4 (2) to show that the polytope has at most eight vertices, so by 1.15 we can use the classification of three-dimensional proper nonsingular toric varieties with Picard number five or less which are minimal in the sense of equivariant blow-ups as described in Oda88, 1.34]. On the other hand if there exists an AS-point, we can use the tools in the previous sections (in particular lemma 1.17(2), prop. 2.2(7) and prop. 3.1) to completely describe the polytope by a suitably generalised notion of a primitive relation.

Recently Kasprzyk classified in Kas03 all 634 three-dimensional terminal polytopes by first describing the minimal cases purely mathematically and then using a computer program for the remaining ones.

\section{Sharp bounds on the number of vertices}

Throughout the section let $P$ be a d-dimensional reflexive polytope in $M_{\mathbb{R}}$.

In higher dimensions only in very special cases classification results exist. So one tries to find at least sharp bounds on invariants and to characterise the case of equality. Here we examine the number of vertices of a reflexive polytope.

This invariant corresponds to the rank of the class group of the associated toric variety $X:=X\left(M, \Sigma_{P}\right)$. There is the exact sequence (see Ful93, Prop. $3.4])$

$$
0 \rightarrow M \rightarrow \operatorname{Div}^{\mathbb{T}}(X) \rightarrow \mathrm{Cl}(X) \rightarrow 0
$$

where $\operatorname{Div}^{\mathbb{T}}(X)$ denotes the free abelian group of torus invariant Weil divisors. Since $\operatorname{Div}^{\mathbb{T}}(X)$ has rank $\left|\Sigma_{P}(1)\right|=|\mathcal{V}(P)|$, we have

$$
\operatorname{rank} \mathrm{Cl}(X)=|\mathcal{V}(P)|-d .
$$

The classification of Kreuzer and Skarke shows that the maximal number of vertices of a $d$-dimensional reflexive polytope is 6 for $d=2,14$ for $d=3$ and 36 for $d=4$. This observation motivates a conjecture:

Definition 5.1. We define $\mathcal{Z}_{2}:=\operatorname{conv}\left( \pm[0,1]^{2}\right)$, it is the (up to isomorphism) unique centrally-symmetric self-dual smooth Fano polytope with 6 vertices (of type $6 a$ in prop. 4.1). We denote by $S_{3}:=X\left(M, \Sigma_{\mathcal{Z}_{2}}\right)$ the associated nonsingular toric Del Pezzo surface that is the blow up of $\mathbb{P}^{2}$ in three torus-invariant points.

Conjecture 5.2. Let $P$ be a d-dimensional reflexive polytope. Then

$$
|\mathcal{V}(P)| \leq 6^{\frac{d}{2}},
$$

where equality occurs if and only if $d$ is even and $P \cong\left(\mathcal{Z}_{2}\right)^{\frac{d}{2}}$.

Remark 5.3. It would be enough to prove this conjecture for $d$ even, because products of reflexive polytopes are again reflexive. 
This conjecture is confirmed by the computer classification for $d \leq 4$, however only for $d=2$ a rigorous proof is known (see cor. 3.3.1) and thm. [5.4(1) below).

The next theorem yields two coarse upper bounds on the number of vertices of a reflexive polytope in terms of some combinatorial invariants of the facets. The first bound is a straightforward generalisation of a bound due to Voskresenskij and Klyachko [VK85, Thm.1] originally proven in the setting of a smooth Fano polytope. The second upper bound is a generalisation of Deb01 Thm. 8], where Debarre improved from a bound of order $O\left(d^{2}\right)$ on the number of vertices of a smooth Fano polytope to a bound of order $O\left(d^{3 / 2}\right)$, which is the asymptotically best upper bound that is known at the moment. We recover the original results for simplicial reflexive polytopes.

Theorem 5.4. Let $P$ be a reflexive polytope.

Define $\alpha:=\max (\mathcal{V}(F): F \in \mathcal{F}(P))$ and $\beta:=\max (\mathcal{F}(F): F \in \mathcal{F}(P))$.

1. $|\mathcal{V}(P)| \leq 2 d \alpha$.

More precisely we distinguish two cases:

If $\alpha \geq 2 d-3$, then $|\mathcal{V}(P)| \leq 2 d(\alpha-d+2)-2$.

If $\alpha \leq 2 d-3$, then $|\mathcal{V}(P)| \leq d \alpha+\alpha-d+1$.

If $P$ is simplicial, i.e., $\alpha=d$, and $d \geq 3$, this yields

$$
|\mathcal{V}(P)| \leq d^{2}+1
$$

2. $|\mathcal{V}(P)| \leq(\alpha-d+1) \beta+2+2 \sqrt{(\alpha-1)(d+1)((\alpha-1)+(\alpha-d+1) \beta)})$. If $P$ is simplicial, i.e., $\alpha=d=\beta$, this yields

$$
|\mathcal{V}(P)| \leq d+2+2 \sqrt{\left(d^{2}-1\right)(2 d-1)} .
$$

Proof. Analysing the proofs of Thm. 1 in VK85 and Thm. 8 in Deb01 in the more general setting of a reflexive polytope, we see that by taking the general invariants $\alpha$ and $\beta$ into account we just have to reprove remark 5(2) in section 2.3 of Deb01, because only there explicitly a lattice basis was used. That result is essentially the first part of the next lemma.

Lemma 5.5. Let $P \subseteq M_{\mathbb{R}}$ be a reflexive polytope.

Let $F \in \mathcal{F}(P), u:=\eta_{F} \in \mathcal{V}\left(P^{*}\right)$ and $\left\{F_{i}\right\}_{i \in I}$ the facets that intersect $F$ in $a(d-2)$-dimensional face. Let $m \in \partial P \cap M$ with $\langle u, m\rangle=0$.

Then $m \in \cup_{i \in I} F_{i}$.

Let additionally $F$ be a simplex with $\mathcal{V}(F)=\left\{e_{1}, \ldots, e_{d}\right\}$. Let $e_{1}^{*}, \ldots, e_{d}^{*}$ be the dual $\mathbb{R}$-basis of $N_{\mathbb{R}}$. For $i=1, \ldots, d$ denote by $F_{i}$ the facet of $P$ such that $F_{i} \cap F=\operatorname{conv}\left(e_{j}: j \neq i\right)$ and choose a lattice point $m^{i}$ on $F_{i}$ that is not contained in $F$.

1. For $i \in\{1, \ldots, d\}$ we have

$$
m \notin F_{i} \Longleftrightarrow\left\langle e_{i}^{*}, m\right\rangle \geq 0 .
$$


2. If there exists $i \in\{1, \ldots, d\}$ such that $m \in F_{i}$ and $m \notin F_{j}$ for all $j \in$ $\{1, \ldots, d\}, j \neq i$, then $m \nsim e_{i}$.

3. If $\left\langle u, m^{i}\right\rangle=0$ and $\left\langle e_{i}^{*}, m^{i}\right\rangle=-1$ for $i=1, \ldots, d-1$, then $e_{1}, \ldots, e_{d}$ is a $\mathbb{Z}$-basis of $M$.

Proof. The first part follows from prop. 2.2 6 ) for $\Pi_{m}$.

Now let $F$ be a simplex. Then $u=\sum_{j=1}^{d}\left(-e_{j}^{*}\right) \in N$. Let $i \in\{1, \ldots, d\}$. Since $m^{i} \notin F$ and 0 is in the interior of $P$, the number $\alpha_{i}:=\frac{-1-\left\langle u, m^{i}\right\rangle}{\left\langle e_{i}^{*}, m^{i}\right\rangle}>0$ is well-defined. We get $\eta_{F_{i}}=u+\alpha_{i} e_{i}^{*}$. From this 1 . is readily derived. 2 . is just a corollary. In 3 . we get $\alpha_{i}=1$ and $e_{i}^{*}=\eta_{F_{i}}-u \in N$ for $i=1, \ldots, d-1$ and $e_{d}^{*}=-\eta_{F_{i}}-e_{1}^{*}-\ldots-e_{d-1}^{*} \in N$.

This proof is inspired by remark 5(2) in section 2.3 of Deb01.

In the following we will focus on the class of simplicial reflexive polytopes, i.e., where the associated varieties are $\mathbb{Q}$-factorial, or equivalently, the class number equals the Picard number. The previous theorem already gave a hint that simplicial reflexive polytopes are actually quite close to smooth Fano polytopes at least when considering only the number of vertices. Also in this case there is an explicit conjecture:

Conjecture 5.6. Let $P$ be a d-dimensional simplicial reflexive polytope. Then

$$
|\mathcal{V}(P)| \leq \begin{cases}3 d & , \quad d \text { even } \\ 3 d-1, & d \text { odd }\end{cases}
$$

For d even equality holds if and only if $P^{*} \cong\left(\mathcal{Z}_{2}\right)^{\frac{d}{2}}$, i.e., $X \cong\left(S_{3}\right)^{\frac{d}{2}}$.

Remark 5.7. It would be enough to prove this conjecture for d even: Assume there were a simplicial reflexive polytope $P$ with $d$ odd and $|\mathcal{V}(P)| \geq 3 d$. Then necessarily $P^{*} \times P^{*} \cong\left(\mathcal{Z}_{2}\right)^{d}$, this would imply $P$ to be centrally symmetric with $|\mathcal{V}(P)|=3 d$, a contradiction to $d$ odd.

The bound is also sharp in the odd-dimensional case, take $X=\mathbb{P}^{1} \times\left(S_{3}\right)^{\frac{d-1}{2}}$. However even for $d=3$ there is exactly one another simplicial reflexive polytope with 8 vertices, it is a smooth Fano polytope, not centrally symmetric, and the associated toric variety $X$ is an equivariant $S_{3}$-fibre bundle over $\mathbb{P}^{1}$.

From Kas03 we get that reflexivity is essential, because the maximal number of vertices a three-dimensional simplicial terminal Fano polytope can have is 10 .

This conjecture was originally proposed in the case of smooth Fano polytopes by Batyrev, and was rigorously proven to hold for (up to) five-dimensional smooth Fano polytopes by Casagrande in Cas03a, Thm. 3.2]. In the above form the conjecture is confirmed by the computer classification of Kreuzer and Skarke for $d \leq 4$. Moreover it yields that there are 194, respectively 5450, classes of three-dimensional, respectively four-dimensional, simplical reflexive polytopes; however only 151 four-dimensional terminal simplicial reflexive polytopes.

The main goal of this section is to give a proof in the case of additional symmetries of the polytope. 
Theorem 5.8. Conjecture 5.6 holds in the case of a simplicial reflexive polytope $P$ where the dual polytope $P^{*}$ contains a vertex $u \in \mathcal{V}\left(P^{*}\right)$ such that $-u \in P^{*}$.

Theorem 5.9. Let $P$ be a centrally symmetric simplicial reflexive polytope. If $d$ is even, then $|\mathcal{V}(P)| \leq 3 d$, with equality iff $P^{*} \cong\left(\mathcal{Z}_{2}\right)^{\frac{d}{2}}$.

If $d$ is odd, then $|\mathcal{V}(P)| \leq 3 d-1$, with equality iff $P^{*} \cong[-1,1] \times\left(\mathcal{Z}_{2}\right)^{\frac{d-1}{2}}$.

We need some preparation for the proofs.

The main result for analysing smooth Fano polytopes is a theorem of Reid about extremal rays of the Mori cone and primitive relations (see Rei83 and Cas03a, Thm. 1.3]). Although for simplicial reflexive polytopes there is no general notion of a primitive relation, for the simplest case as defined in prop. 3.1 we still have an analogous result (recall definition 2.1):

Lemma 5.10. Let $P$ be a simplicial reflexive polytope.

Let $v \in \mathcal{V}(P), w \in \partial P \cap M$ with $v+w \in \partial P$ and $z:=z(v, w)$.

Let $x \in \partial P, x \notin\{v, w, z\}$, with $x \sim z$ and $x$ away from $v$.

Then $\operatorname{conv}(x, z, w)$ is contained in a face.

Moreover exactly one of the following two conditions holds:

1. Any facet containing $x$ and $z$ contains also $w$.

2. There exists a facet $F$ with $x, v, z \in F$.

The second case must occur, if $w \in \mathcal{V}(P)$ and $x$ is away from $w$.

If the second case occurs, we have:

For any such $F$ there exists a unique facet $G$ with $x, w, z \in G$ such that $F \cap G$ is a $(d-2)$-dimensional face of $P . F \cap G$ consists of those elements of $F$ that are away from $v$, respectively those elements of $G$ that are away from the (unique) vertex not in $F$. Obviously $w \notin F$ and $v \notin G$.

Proof. Assume the first case is not fulfilled. Prop. 3.1 (ii) implies that there exists a facet $F \in \mathcal{F}(P)$ with $x, z, v \in F$. By [3.1 (iii) there is a facet $G \in \mathcal{F}(P)$ containing $w$ and $z$ such that $F \cap G$ is a $(d-2)$-dimensional face. Since $F, G$ are simplices and $v \in \mathcal{V}(F)$, the remaining statements are now straightforward.

The next result is a generalisation of a lemma proven by Casagrande Cas03a Lemma 2.3] for smooth Fano polytopes, here we recover the original statement in the more general setting of a terminal simplicial reflexive polytope.

Lemma 5.11. Let $P$ be a simplicial reflexive polytope.

Let $v, w \in \mathcal{V}(P), w^{\prime} \in \partial P \cap M$ away from $w$. Furthmore let $v+w \in \partial P$ and $v+w^{\prime} \in \partial P, z:=z(v, w)$ and $z^{\prime}:=z\left(v, w^{\prime}\right)$.

We define $K:=P \cap \operatorname{lin}\left(v, w, w^{\prime}\right)$. Then $K$ is a two-dimensional reflexive polytope (of possible types $5 a, 6 a, 6 b, 7 a$ in prop. 4.1).

If $K$ is terminal, then $z=v+w, w^{\prime}=-v-w=-z, z^{\prime}=v+w^{\prime}=-w$; and either $\partial K \cap M=\left\{v, w, z, w^{\prime}, z^{\prime}\right\}$ or $\partial K \cap M=\left\{v, w, z, w^{\prime}, z^{\prime},-v=w+w^{\prime}=\right.$ $\left.z\left(w, w^{\prime}\right)\right\}$.

Proof. Let $z=a v+b w$ and $z^{\prime}=a^{\prime} v+b^{\prime} w^{\prime}$ as in 3.1. We note that $w^{\prime}$ and $z$ is away from $v$ and $w ; z^{\prime}$ is away from $v$ and $w^{\prime}$. 
Assume $w^{\prime} \sim z$. Since $w^{\prime}$ is away from $w \in \mathcal{V}(P)$, it follows from 5.10 that there exists a facet that contains $w^{\prime}, z, v$, hence $w^{\prime} \sim v$, a contradiction.

Thus $w^{\prime} \nsim z$.

There are now two different cases, and it must be shown that the second one cannot occur.

1. $v, w, w^{\prime}$ are linearly dependent.

By 3.1 there are three possibilities:

If $w \sim w^{\prime}$, then $K=\operatorname{conv}\left(v, z, w, z^{\prime}, w^{\prime}\right)$. If $w+w^{\prime}=0$, then $v \in \operatorname{conv}(v+$ $\left.w, v+w^{\prime}\right)$, a contradiction. If $v+w \in \partial P$, then $K=\operatorname{conv}\left(v, z, w, z^{\prime}, w^{\prime}\right.$, $\left.z\left(w, w^{\prime}\right)\right)$.

Thus in any case $K$ is a lattice polytope with 5 or 6 vertices, canonical, hence by 1.17(1) reflexive. By analysing the cases in 4.1 we get the remaining statements.

2. $v, w, w^{\prime}$ are linearly independent.

Hence also $z, z^{\prime}, v$ are linearly independent.

Assume $z^{\prime} \sim z$. 5.10 implies that $\operatorname{conv}\left(z^{\prime}, z, w\right)$ is contained in a facet $F \in \mathcal{F}(P)$. Since $v \notin F$ and $z^{\prime} \in F$, 3.1 (ii) implies $w^{\prime} \in F$, a contradiction to $w^{\prime} \nsim z$.

Thus $z \not z^{\prime}$.

By assumption $z+z^{\prime} \neq 0$, hence $z+z^{\prime} \in \partial P$. Let $y:=z\left(z^{\prime}, z\right)=$ $k z^{\prime}+l z \in \partial P \cap M$. We have $y \notin\left\{z, z^{\prime}, v, w, w^{\prime}\right\}$, because $v, w, w^{\prime}$ are linearly independent. Choose $y^{\prime} \in \partial P$ with $y^{\prime}=v+\lambda(y-v)$ for $\lambda \geq 1$ maximal, so that $y^{\prime}$ is away from $v$. Furthermore $y^{\prime} \sim z^{\prime}$ and $y^{\prime} \sim z$. So by 5.10 there exist facets $F_{1}, F_{2} \in \mathcal{F}(P)$ such that $\operatorname{conv}\left(y^{\prime}, z, w\right) \subseteq F_{1}$ and $\operatorname{conv}\left(y^{\prime}, z^{\prime}, w^{\prime}\right) \subseteq F_{2} ; v \notin F_{1}, F_{2}$.

Now choose $y^{\prime \prime}=w+\mu\left(y^{\prime}-w\right) \in P$ for $\mu \geq 1$ maximal; so $y^{\prime \prime}$ is away from $w$. Furthermore $\operatorname{conv}\left(y^{\prime \prime}, y^{\prime}, z, w\right) \subseteq F_{1}$ and $y^{\prime \prime}$ away from $v$, so by 5.10 there exists a facet $G \in \mathcal{F}(P)$ that contains $y^{\prime \prime}, v, z$ and intersects $F_{1}$ in a $(d-2)$-dimensional face. Hence necessarily $\left\langle\eta_{F_{1}}, v\right\rangle=\frac{b-1}{a}$ and $\left\langle\eta_{G}, w\right\rangle=\frac{a-1}{b}$.

$K$ is a three-dimensional polytope. Any face of $K$ is contained in a face of $P$. We have $F_{1} \neq F_{2}$, since $w^{\prime} \nsim z$. So $C:=F_{1} \cap F_{2} \cap K$ is a vertex or edge of $K$ containing $y^{\prime}$. Since also $w^{\prime}+z \neq 0$ and $w^{\prime} \not z$, we get $w^{\prime}+z \in \partial P$. We set $x:=z\left(w^{\prime}, z\right) \in \partial P \cap M$.

We distinguish several cases:

(a) $y^{\prime}=y$.

i. $w \in F_{2}$.

The vertices of $C$ consist of $y^{\prime \prime}$ and $w$. It is also easily seen that $x \in C$ with $w \neq x \neq y$, so also $x \neq y^{\prime \prime}$. If $a=1$, it were $0=\left\langle\eta_{G}, w\right\rangle>\left\langle\eta_{G}, x\right\rangle>\left\langle\eta_{G}, y^{\prime \prime}\right\rangle=-1$, a contradiction.

Therefore $a \geq 2, b=1,\left\langle\eta_{G}, w\right\rangle=a-1>0$.

Then $\left\langle\eta_{F_{2}}, v\right\rangle=\frac{b^{\prime}-1}{a^{\prime}}$ and $-1=\left\langle\eta_{F_{2}}, y\right\rangle=-k+l a \frac{b^{\prime}-1}{a^{\prime}}-l b$, thus, since $b=1$,

$$
\frac{k-1}{l}=a \frac{b^{\prime}-1}{a^{\prime}}-1 \in \mathbb{N} .
$$


Since $\frac{b^{\prime}-1}{a^{\prime}} \neq 0, b^{\prime} \geq 2$ and $a^{\prime}=1$.

If $k=1$, then (4) yields $1=a\left(b^{\prime}-1\right) \geq a \geq 2$, a contradiction.

If $l=1$, then $\left\langle\eta_{G}, y\right\rangle=-k-1+k b^{\prime}\left\langle\eta_{G}, w^{\prime}\right\rangle$. So $-1=\left\langle\eta_{G}, y^{\prime \prime}\right\rangle=$

$(1-\mu)(a-1)+\mu\left\langle\eta_{G}, y\right\rangle=(1-\mu)(a-1)-\mu k-\mu+\mu k b^{\prime}\left\langle\eta_{G}, w^{\prime}\right\rangle$.

This implies $\left\langle\eta_{G}, w^{\prime}\right\rangle=\frac{\frac{-a}{\mu}+k+a}{k b^{\prime}}$. Since (44) yields $k=a\left(b^{\prime}-1\right)$, this implies

$$
\left\langle\eta_{G}, w^{\prime}\right\rangle=\frac{\frac{-1}{\mu b^{\prime}}+1}{b^{\prime}-1} \in \mathbb{N} .
$$

On the other hand $\mu \geq 1$ and $b^{\prime} \geq 2$ yields $0<\frac{-1}{\mu b^{\prime}}+1<1$, this contradicts the previous equation.

ii. $w \notin F_{2}$.

This immediately implies $y^{\prime \prime}=y$. We find $x^{\prime} \in F_{1}$ such that $x \in\left[w, x^{\prime}\right]$ and $x^{\prime}$ is away from $w$. By 5.10 we have $x^{\prime} \in F_{1} \cap G$. As $w^{\prime} \nsim z$, we obtain $x^{\prime}=y$. Hence $\left.x \in\right] w, y[$.

Since $w^{\prime} \sim x$ there exists a facet $H \in \mathcal{F}(P)$ containing $w^{\prime}, w, y$; furthermore $H \neq F_{2}$, since $w \notin F_{2}$. Hence there are edges $F_{2} \cap$ $H \supseteq\left[w^{\prime}, y\right]$ and $F_{1} \cap H=[w, y]$ of $K$.

Since $w \not z^{\prime}$ we can define in a double recursion $x_{r}^{0}:=x$, $x_{l}^{0}:=x\left(z^{\prime}, w\right), x_{r}^{i}:=x\left(x_{l}^{i-1}, z\right), x_{l}^{i}:=x\left(z^{\prime}, x_{r}^{i-1}\right)$ for $i \in \mathbb{N}$, $i \geq 1$. As $w^{\prime}, y, z$ and $w, y, z^{\prime}$ are linearly independent, we easily see that this procedure is well-defined, and $x_{l}^{0}, x_{l}^{1}, x_{l}^{2}, \ldots$ are pairwise different lattice points in $] w^{\prime}, y\left[\right.$ and $x_{r}^{0}, x_{r}^{1}, x_{r}^{2}, \ldots$ are pairwise different lattice points in $] w, y[$. Hence we have constructed infinitely many lattice points in $P$, a contradiction.

(b) $y^{\prime} \neq y$.

If $y^{\prime \prime} \neq y^{\prime}$, then obviously $y$ is a lattice point in the interior of $K$, a contradiction.

Thus $y^{\prime \prime}=y^{\prime}$. This implies $\left.y \in\right] v, y^{\prime}\left[\right.$, so $y \in G$, hence $\operatorname{conv}\left(z^{\prime}, y^{\prime}, y, v\right)$ is contained in a facet $F^{\prime} \in \mathcal{F}(P)$. 5.10 implies that there exists a unique facet $G^{\prime} \in \mathcal{F}(P)$ that contains $w^{\prime}, z^{\prime}, y^{\prime}$ such that $F^{\prime} \cap G^{\prime}$ is a $(d-2)$-dimensional face.

Furthermore $\frac{b-1}{a}=\left\langle\eta_{F_{1}}, v\right\rangle>\left\langle\eta_{F_{1}}, y\right\rangle>\left\langle\eta_{F_{1}}, y^{\prime}\right\rangle=-1$, hence $b \geq 2$ and $a=1$. Especially we get $\left\langle\eta_{G}, w\right\rangle=0$.

This yields again $x \in F_{1}$.

i. $w \in G^{\prime}$.

Since $w \in \mathcal{V}(P), w \notin F^{\prime}$ and $w^{\prime}$ is away from $w \in \mathcal{V}\left(G^{\prime}\right)$, 5.10 implies that $w^{\prime} \in G^{\prime} \cap F^{\prime}$, a contradiction.

ii. $w \notin G^{\prime}$.

Let $C^{\prime}:=G^{\prime} \cap F_{1} \cap K$. Then $y^{\prime}$ is a vertex of $C^{\prime}$.

Assume $C^{\prime}$ were an edge. Let $v^{\prime} \in \mathcal{V}\left(C^{\prime}\right)$ with $v^{\prime} \neq y^{\prime}$. This implies $v^{\prime} \neq w$. Then $v^{\prime}$ is away from $w$, hence by [5.10 $v^{\prime} \in G$, therefore $v^{\prime}=y^{\prime}$, a contradiction.

So $C^{\prime}=\left\{y^{\prime}\right\}$, and just the same way we see that $\left[y^{\prime}, w\right]$ is an edge of $K$. Therefore $\left.x \in] w, y^{\prime}\right]$. Since $\left.0=\left\langle\eta_{G}, w\right\rangle\right\rangle\left\langle\eta_{G}, x\right\rangle \geq$ $\left\langle\eta_{G}, y^{\prime}\right\rangle=-1$, this implies $x=y^{\prime}$.

Furthermore we have $y^{\prime}=(1-\lambda) v+\lambda y=\left((1-\lambda)+\lambda\left(k a^{\prime}+\right.\right.$ $l a)) v+\lambda k b^{\prime} w^{\prime}+\lambda l b w$, where $\lambda>1$. On the other hand $x=$ $r w^{\prime}+s z=r w^{\prime}+s a v+s b w$ for $r, s \in \mathbb{N}, r, s \geq 1$. 
Comparing the coefficients for $w^{\prime}$ and $w$ this yields

$$
\lambda l=s, \quad \lambda k b^{\prime}=r .
$$

From the first equation we get $s=\lambda l>l \geq 1$, so $s \geq 2$. This implies $1=r=\lambda k b^{\prime}>k b^{\prime} \geq 1$, a contradiction.

Using prop. 2.2(1-4) and analysing the possible cases in prop. 4.1 it is straightforward to prove a corollary of the previous lemma:

Corollary 5.12. Let $P$ be a simplicial reflexive polytope and $v \in \mathcal{V}(P)$.

There are at most three vertices of $P$ not in the star set of $v$; equality implies that $-v \in \mathcal{V}(P)$. For $P_{v}:=\Pi_{v}(P)$ and $M_{v}:=M / \mathbb{Z} v$ we have

$$
|\mathcal{V}(P)| \leq\left|\partial P_{v} \cap M_{v}\right|+4,
$$

where equality implies $-v \in \mathcal{V}(P)$. There are now two cases:

1. Let $w \in \mathcal{V}(P)$ with $w \neq-v$ and $w \nsim v$.

Then any lattice point on the boundary of $P$ is in the star set of $v$ or in the star set of $w$ but not away from $w$ or in $\operatorname{lin}(v, w)$. This implies

$\left|P_{v} \cap M_{v}\right|+\left|\operatorname{int} P_{w} \cap M_{w}\right| \leq|\partial P \cap M| \leq\left|P_{v} \cap M_{v}\right|+\left|\operatorname{int} P_{w} \cap M_{w}\right|+2 ;$

if the second equality holds, then $-v \in P$.

2. No such $w$ as in 1. exists. Then:

$$
|\mathcal{V}(P)| \leq\left|\partial P_{v} \cap M_{v}\right|+2 .
$$

Going back to algebraic geometry we derive a generalisation of a theorem proven by Casagrande in the nonsingular case Cas03a, Thm. 2.4]:

Corollary 5.13. If $X$ is a $\mathbb{Q}$-factorial Gorenstein toric Fano variety with torusinvariant prime divisor $\mathcal{V}_{\tau}$, then the Picard numbers satisfy the inequality

$$
\rho_{X}-\rho \mathcal{V}_{\tau} \leq 3
$$

Finally using lemmas 5.5 and 5.11 we are now ready to prove the main theorems.

Proof of theorems 5.8 and 5.9. Let $P$ be a simplicial reflexive polytope such that there exists a vertex $u \in \mathcal{V}\left(P^{*}\right)$ with $-u \in P^{*}$. Let $F$ be the facet corresponding to $u$ and $F^{\prime}$ the face defined by $-u$. Now define the set $\left\{v^{1}, \ldots, v^{d}\right\}$ of vertices not in $F$ but in facets intersecting $F$ in a codimension two face. Lemma 5.5 immediately implies that $\mathcal{V}(P) \backslash\left(\mathcal{V}(F) \cup \mathcal{V}\left(F^{\prime}\right)\right)=\{v \in \mathcal{V}(P):\langle u, v\rangle=0\} \subseteq$ $\left\{v^{1}, \ldots, v^{d}\right\}$. This yields the bound $|\mathcal{V}(P)| \leq 3 d$. Now we must consider two special cases: 
1. In order to prove conjecture [5.6] and thereby finish the proof of theorem 5.8 we may assume that $|\mathcal{V}(P)|=3 d$ and $d$ is even by remark 5.7 Then 5.5 implies that $\{x \in \partial P \cap M:\langle u, x\rangle=0\}=\left\{v^{1}, \ldots, v^{d}\right\}$ is a set of cardinality $d$, and also $F^{\prime}$ must contain $d$ vertices, $F^{\prime}$ is therefore a $d-1$ dimensional simplex, so $-u \in \mathcal{V}\left(P^{*}\right)$.

Let $e_{1}, \ldots, e_{d}$ be the vertices of the facet $F$, and $b_{1}, \ldots, b_{d}$ the vertices of $F^{\prime}$ such that $v^{1}, \ldots, v^{d}$ are exactly the corresponding vertices analogously constructed as in 5.5 Define also $F_{i}:=\operatorname{conv}\left(v^{i}, e_{j}: j \neq i\right)$ for $i=$ $1, \ldots, d$. Then we get the following three facts for $i, j, k \in\{1, \ldots, d\}$ :

- Fact 1: For $i \neq j: v^{i} \sim v^{j}$ or $v^{i}+v^{j}=0$.

(Proof: Assume not. Then there exists a $k$ such that $v^{i}+v^{j}=v^{k} \in F_{k}$. By 3.11(ii) this implies $v^{i} \in F_{k}$ or $v^{j} \in F_{k}$, giving $v^{i}=v^{k}$ or $v^{j}=v^{k}$, a contradiction.)

- Fact 2: For $i: e_{i}+v^{i} \in \partial P$ and $b_{i}+v^{i} \in \partial P$.

(Proof: Since $v^{i} \notin F_{j}$ for all $j \neq i$ and $v^{i} \in F_{i}$, [5.5(2) yields $e_{i} \nsim v^{i}$. By symmetry the same holds for $b_{i}$.)

- Fact 3: Let $i, j$ such that $e_{i}+b_{j} \in \partial P$. Then $z\left(e_{i}, b_{j}\right)=e_{i}+b_{j}=v^{k}$ for some $i \neq k \neq j$.

(Proof: Since $\left\langle u, e_{i}+b_{j}\right\rangle=0$, let $e_{i}+b_{j}=v^{k}$ for some $k$. Assume $e_{i} \nsim v^{k}$. By 3.1 then also $2 e_{i}+b_{j} \in \partial P$. This implies $v^{k}=1 / 2\left(b_{j}+\left(2 e_{i}+b_{j}\right)\right)$, a contradiction to $v^{k} \in \mathcal{V}(P)$. By symmetry we get $e_{i} \sim v^{k} \sim b_{j}$. By fact 2 necessarily $i \neq k \neq j$.)

Let $i \in\{1, \ldots, d\}$. By fact 2 we can apply lemma 5.11 to the vertices $v^{i}, e_{i}, b_{i}$. From fact 3 and analysing the possible types in 4.1 we get that $P \cap \operatorname{lin}\left(v^{i}, e_{i}, b_{i}\right)$ must be a terminal two-dimensional reflexive polytope, so $-e_{i}=v^{i}+b_{i} \in F^{\prime},-b_{i}=v^{i}+e_{i} \in F, v^{i}=z\left(-e_{i},-b_{i}\right)=-e_{i}+\left(-b_{i}\right)$. As this is true for all $i=1, \ldots, d$, we get $F^{\prime}=-F$ and $-e_{i},-b_{i} \in \mathcal{V}(P)$. This gives a map

$$
s:\{1, \ldots, d\} \rightarrow\{1, \ldots, d\}, i \mapsto s(i), \text { such that } b_{s(i)}:=-e_{i} .
$$

(a) $s$ is injective, hence a permutation.

(b) There are no fixpoints under $s$, i.e., $s(i) \neq i$ for all $\{1, \ldots, d\}$.

(c) $-v^{i}=e_{i}+b_{i} \in \partial P$ for all $i \in\{1, \ldots, d\}$.

(Proof: By 5.11 it is enough to show that $-v^{i} \in P$. Assume not. Fact 1 implies then $v^{i} \sim v^{s(i)}=z\left(-e_{s(i)},-b_{s(i)}\right)=z\left(-e_{s(i)}, e_{i}\right)$, so by [5.10] $v^{i} \sim e_{i}$, a contradiction to fact 2.)

(d) $s \circ s=$ id.

(Proof: Assume there exists an $i \in\{1, \ldots, d\}$ such that for $j:=s(i)$ we have $b_{s(j)} \neq b_{i}$. This implies $b_{i} \sim v^{s(i)}=z\left(-e_{s(i)},-b_{s(i)}\right)=$ $z\left(b_{s(j)}, e_{i}\right)$, so by assumption and $5.10 b_{i} \sim e_{i}$. This is a contradiction to $(\mathrm{c})$.)

Property (d) implies that $P$ is centrally symmetric. Furthermore $s$ is a product of $\frac{d}{2}$ disjoint transpositions in the symmetric group of $\{1, \ldots, d\}$. This permutation $s$ and the set $\left\{e_{1}, \ldots, e_{d}\right\}$ of vertices of $F$ uniquely determine $P$, because $F^{\prime}=-F$ and $v^{i}=-e_{i}+e_{s(i)}$ for all $i \in\{1, \ldots, d\}$. 
For any $i \in\{1, \ldots, d\}$ we get $\left\langle u, v^{i}\right\rangle=0$ and $\left\langle e_{i}^{*}, v^{i}\right\rangle=\left\langle e_{i}^{*},-e_{i}+e_{s(i)}\right\rangle=$ -1 . Hence 5.5 (3) implies that $e_{1}, \ldots, e_{d}$ is a $\mathbb{Z}$-basis of $M$. This immediately yields the uniqueness of $P$ up to isomorphism of the lattice.

2. To finish the proof of theorem 5.9 let $|\mathcal{V}(P)|=3 d-1, d$ odd and $P$ be centrally symmetric. Then $-u \in \mathcal{V}\left(P^{*}\right)$ and $F^{\prime}=-F$. By [5.5] we can assume that $\{x \in \mathcal{V}(P) \cap M:\langle u, x\rangle=0\}=\left\{v^{1}, \ldots, v^{d-1}\right\}$ is a set of cardinality $d-1$. We can again enumerate the vertices of $F$ as $e_{1}, \ldots, e_{d}$ such that $v^{i}$ is a facet of $F_{i}:=\operatorname{conv}\left(v^{i}, e_{j}: j \neq i\right)$ for $i=1, \ldots, d-1$. There is a map

$$
\sigma:\{1, \ldots, d-1\} \rightarrow\{1, \ldots, d-1\}, \quad i \mapsto \sigma(i), \text { such that } v^{\sigma(i)}:=-v^{i} .
$$

$\sigma$ is again just a product of $\frac{d-1}{2}$ disjoint transpositions. Distinguish two cases:

(a) $\left\langle u, v^{d}\right\rangle=0$.

So there exists exactly one $k \in\{1, \ldots, d-1\}$ such that $v^{d}=v^{k}$. We may assume $k=d-1$ and $\sigma(d-1)=d-2$. From [5.5(2) and since $v^{d-2} \in \mathcal{V}(P)$ we get $y:=v^{d-2}+e_{d-2}=z\left(v^{d-2}, e_{d-2}\right) \in F$, hence $e_{d-2}=y+v^{d-1}=z\left(y, v^{d-1}\right)$. For $j=1, \ldots, d-3$ we have $e_{d-2} \in F_{j}$ and $v^{d-1} \notin F_{j}$, hence $y \in F_{j}$ by 3.1 (ii). Since $y$ is away from $e_{d-2}$, this implies $y \in\left[e_{d-1}, e_{d}\right]$. As $e_{d-1} \sim v^{d}=v^{d-1} \sim e_{d}$ and $v^{d-1} \nsim y$, it follows that $y \notin\left\{e_{d-1}, e_{d}\right\}$, hence $\left.y \in\right] e_{d-1}, e_{d}\left[\right.$. Now $e_{d} \sim v^{d-1}=$ $-y+e_{d-2}=z\left(-y, e_{d-2}\right)$, so by [5.10] also $\left.e_{d} \sim-y \in\right]-e_{d-1},-e_{d}[$. This implies $e_{d} \sim\left(-e_{d}\right)$, a contradiction.

(b) $\left\langle u, v^{d}\right\rangle \neq 0$.

Therefore $v^{d} \in \mathcal{V}(-F)$. Since $v^{d} \sim e_{i}$ for $i=1, \ldots, d-1$, we have $v^{d}=-e_{d}$. For $i=1, \ldots, d-1$ lemma 5.5(2) implies again $v^{i}+e_{i} \in$ $\partial P$, so by central symmetry also $v^{i}-e_{\sigma(i)} \in \partial P$. Using [5.11] we derive $v^{i}=e_{\sigma(i)}-e_{i}$. Especially $\left\langle u, v^{i}\right\rangle=0$ and $\left\langle e_{i}^{*}, v^{i}\right\rangle=-1$, hence [5.5] (3) implies that $e_{1}, \ldots, e_{d}$ is a $\mathbb{Z}$-basis of $M$. This yields the uniqueness of $P$, hence $P \cong\left([-1,1] \times\left(S_{3}\right)^{\frac{d-1}{2}}\right)^{*}$.

\section{Sharp bounds on the number of lattice points}

Throughout the section let $P$ be a d-dimensional Fano polytope in $M_{\mathbb{R}}$.

If $P$ is reflexive, then the number of lattice points in $P$ is $h^{0}\left(X^{*},-K_{X^{*}}\right)$ for $X^{*}:=X\left(N, \mathcal{N}_{P}\right)$ (see [Ful93, 3.4]). In general there exist very large explicit bounds on $|\partial P \cap M|$ for the class of canonical Fano polytopes (see [ZL91]). However in some cases we can simply count integral points modulo $k$, a method that is originally due to Batyrev (see [Bat82, Lemma 1]).

Definition 6.1. For $k \in \mathbb{N}$ we have the canonical homomorphism

$$
\alpha_{k}: M \rightarrow M / k M \cong(\mathbb{Z} / k \mathbb{Z})^{d} .
$$


For a convex set $C \subseteq M_{\mathbb{R}}$ with $C \cap M \neq \emptyset$ one easily sees that the minimal $k \in \mathbb{N}_{\geq 1}$ such that the restriction of $\alpha_{k}$ to $C \cap M$ is injective is just the maximal number of lattice points on an intersection of $C$ with an affine line. This invariant minus one is called the discrete diameter of $C$ in Kan98.

Lemma 6.2. Let $d \geq 2, P$ a canonical Fano polytope and $B \subseteq \partial P \cap M$ with $|[x, y] \cap M|=2$ for all $x, y \in B, x \neq y, x \sim y$. Let $s$ denote the number of centrally symmetric pairs in $B$. Then

$$
|B| \leq 2^{d+1}-2, \quad s \geq|B|+1-2^{d} .
$$

Proof. We consider the restriction of $\alpha_{2}$ to $B$. As $P$ is canonical, the fibre of 0 is empty. Using the assumption it is also easy to see that the fibre of a non-zero element in $(\mathbb{Z} / 2 \mathbb{Z})^{d}$ has at most two elements, and in the case of equality it consists of one pair of centrally symmetric lattice points in $B$. From this the bounds can be derived.

We immediately get a sharp bound on the number of vertices of a semiterminal canonical Fano polytope (see def. [2.3). In particular we get a result that was proven in the case of a smooth Fano polytope in [Bat99, Prop. 2.1.11]:

Corollary 6.3. Let $P$ be a terminal Fano polytope. Then

$$
|\partial P \cap M|=|\mathcal{V}(P)| \leq 2^{d+1}-2 .
$$

If equality holds, then $P$ is centrally symmetric. This holds for the terminal reflexive $d$-dimensional standard lattice zonotope $\mathcal{Z}_{d}:=\operatorname{conv}\left( \pm[0,1]^{d}\right)$, see [DHZ01, Proof of thm. 3.21] for $\mathcal{Z}^{(d)}=\mathcal{Z}_{d-1}$.

The results in Kas03 show that $\mathcal{Z}_{d}$ is even the only terminal Fano polytope with the maximal number of vertices for $d \leq 3$. However the computer classification of Kreuzer and Skarke yields two non-isomorphic four-dimensional terminal reflexive polytopes with $2^{5}-2=30$ vertices.

The second case where counting modulo $k$ works is the class of centrally symmetric reflexive polytopes. In the case of a smooth Fano polytope where centrally symmetric pairs of vertices span $M_{\mathbb{R}}$ there exists a complete explicit classification that is due to Casagrande (see Cas03b). However we cannot expect such a result for centrally symmetric reflexive polytopes, since by the classification of Kreuzer and Skarke there are 150 centrally symmetric reflexive polytopes already in dimension four. For $d=2$ we have 3 (see 4.1) and for $d=3$ there are 13 (see Wag95) $d$-dimensional centrally symmetric reflexive polytopes.

There is the conjecture due to Ewald (see [Ewa88]) that any d-dimensional smooth Fano polytope can be embedded in the unit lattice cube $[-1,1]^{d}$. It is proven for $d \leq 4$ by the classification or under additional symmetries. It is wrong for simplicial reflexive polytopes, e.g., type 9 in prop. 4.1 contains 10 lattice points.

However for $d \leq 3$ we can always embedd a centrally symmetric reflexive polytope $P$ in the unit lattice cube. For this we choose by $1.17(1,2)$ a $\mathbb{Z}$-basis 
$b_{1}, \ldots, b_{d}$ of lattice points in $\partial P^{*}$, so $P \subseteq\left\{\sum \lambda_{i} b_{i}^{*}: \lambda_{i} \in\{-1,0,1\}\right\}$ for the dual $\mathbb{Z}$-basis $b_{1}^{*}, \ldots, b_{d}^{*}$.

There is no such result for $d \geq 4$. For instance let $P$ be the 4 -dimensional centrally symmetric reflexive polytope $P$ in example [2.6 Assume $P^{*}$ could be embedded as a lattice polytope in $[-1,1]^{4}$. Then $\left([-1,1]^{4}\right)^{*}$ would be a lattice subpolytope of $P$ with the same number of vertices. Since $P$ is terminal, this would be an equality, a contradiction. This example is taken from Wir97 where this topic is thoroughly dicussed. In Wir97 there is also a characterisation of centrally symmetric reflexive polytopes presented that have the minimal number of $2 d$ vertices, and it is shown that these polytopes can always be embedded in the unit lattice cube.

By such an embedding we trivially get that the number of lattice points in the polytope is bounded by $3^{d}$ with equality only in the case of the unit lattice cube. However this is even true in general:

Theorem 6.4. Let $P$ be a centrally symmetric canonical Fano polytope. Then

$$
|P \cap M| \leq 3^{d} .
$$

Any facet of $P$ has at most $3^{d-1}$ lattice points. If $P$ is additionally reflexive, then the following statements are equivalent:

1. $|P \cap M|=3^{d}$

2. Every facet of $P$ has $3^{d-1}$ lattice points

3. $P \cong[-1,1]^{d}$ as lattice polytopes

Proof. For the bounds we just have to show that $\left.\alpha_{3}\right|_{P \cap M}$ is injective. So suppose there are $x, y \in P \cap M$ such that $\alpha_{3}(x)=\alpha_{3}(y)$. This implies $(y-x) / 3 \in$ $\operatorname{int} P \cap M=\{0\}$, hence $x=y$.

The equivalences in the case of a reflexive polytope will be proven in $\mathrm{Nil04}$.

\section{References}

[AKMS97] Avram, A.C.; Kreuzer, M.; Mandelberg, M.; Skarke, H.: Searching for K3 Fibrations. Nucl. Phys. B 494, 567-589 (1997)

[Bat82] Batyrev, V.V.: Toroidal Fano 3-folds. Math. USSR-Izv. 19, 13-25 (1982)

[Bat91] Batyrev, V.V.: On the classification of smooth projective toric varieties. Tohoku Math. J. 43, 569-585 (1991)

[Bat94] Batyrev, V.V.: Dual polyhedra and mirror symmetry for CalabiYau hypersurfaces in toric varieties. J. Algebr. Geom. 3, 493-535 (1994)

[Bat99] Batyrev, V.V.: On the classification of toric Fano 4-folds. J. Math. Sci., New York 94, 1021-1050 (1999) 
[Bor00] Borisov, A.: Convex lattice polytopes and cones with few lattice points inside, from a birational geometry viewpoint. Preprint, math.AG/0001109 (2000)

[Cas03a] Casagrande, C.: Toric Fano varieties and birational morphisms. Int. Math. Res. Not. 27, 1473-1505 (2003)

[Cas03b] Casagrande, C.: Centrally symmetric generators in toric Fano varieties. Manuscr. Math. 111, No.4, 471-485 (2003)

[Con02] Conrads, H.: Weighted projective spaces and reflexive polytopes. Manuscripta Math. 107, 215-227 (2002)

[Deb01] Debarre, O.: Toric Fano varieties. In: Higher dimensional varieties and rational points, lectures of the summer school and conference, Budapest 2001. Bolyai Society Mathematical Studies 12, pages 93132. Berlin: Springer 2001

[DHZ01] Dais, D.I.; Haase, C.; Ziegler, G.M.: All toric l.c.i.-singularities admit projective crepant resolutions. Tohoku Math. J. 53, 95-107 (2001)

[Ewa88] Ewald, G.: On the classification of toric Fano varieties. Discrete Comput. Geom. 3, 49-54 (1988)

[Ewa96] Ewald, G.: Combinatorial convexity and algebraic geometry. Graduate texts in mathematics 168. New York: Springer 1996

[Ful93] Fulton, W.: Introduction to toric varieties. Annals of Mathematics Studies 131. Princeton, NJ: Princeton University Press 1993

[Kan98] Kantor, J.-M.: Triangulations of integral polytopes and Ehrhart polynomials. Contrib. to Alg. and Geom. 39, 205-218 (1998)

[Kas03] Kasprzyk, A.M.: Toric Fano 3-folds with terminal singularities. Preprint, math.AG/0311284 (2003)

[KS97] Kreuzer, M.; Skarke, H.: On the classification of reflexive polyhedra. Commun. Math. Phys. 185, 495-508 (1997)

[KS98] Kreuzer, M.; Skarke, H.: Classification of reflexive polyhedra in three dimensions. Adv. Theor. Math. Phys. 2, 853-871 (1998)

[KS00a] Kreuzer, M.; Skarke, H.: Complete classification of reflexive polyhedra in four dimensions. Adv. Theor. Math. Phys. 4, 1209-1230 (2000)

[KS00b] Kreuzer, M.; Skarke, H.: Reflexive polyhedra, weights and toric Calabi-Yau fibrations. Rev. Math. Phys. 14, 343-374 (2002)

[KS02] Kreuzer, M.; Skarke, H.: PALP: A package for analyzing lattice polytopes with applications to toric geometry. Preprint, math.NA/0204356 (2002)

[Nil04] Nill, B.: Toric Fano varieties with reductive group of automorphisms. In preparation (2004) 
[Oda88] Oda, T.: Convex bodies and algebraic geometry - An introduction to the theory of toric varieties. Ergebnisse der Mathematik und ihrer Grenzgebiete 15. Berlin: Springer 1988

[PR00] Poonen, B.; Rodriguez-Villegas, F.: Lattice polygons and the number 12. Am. Math. Soc. Monthly 107, 238-250 (2000)

[Rei83] Reid, M.: Decomposition of toric morphisms. In: Arithmetic and geometry, Vol. II: Geometry. Progress in Mathematics 36, pages 395-418. Boston: Birkhäuser 1983

[Sat00] Sato, H.: Toward the classification of higher-dimensional Fano varieties. Tohoku Math. J., 52, 383-413 (2000)

[Sti01] Stillwell, J.: The story of the 120-cell. Notices of the Am. Math. Soc. 48, 17-24 (2001)

[VK85] Voskresenskij, V.E.; Klyachko A.A.: Toroidal Fano varieties and root systems. Math. USSR-Izv. 24, 221-244 (1985)

[Wag95] Wagner, H.: Gewichtete projektive Räume und reflexive Polytope. Diplomarbeit (in German). Bochum: Math. Inst. der RuhrUniversität Bochum 1995

[Wir97] Wirth, P.R.: Zentralsymmetrische reflexive Polytope. Diplomarbeit (in German). Bochum: Math. Inst. der Ruhr-Universität Bochum 1997

[WW82] Watanabe, K.; Watanabe, M: The classification of Fano 3-folds with torus embeddings. Tokyo J. Math. 5, 37-48 (1982)

[ZL91] Ziegler, G.M.; Lagarias, J.C.: Bounds for lattice polytopes containing a fixed number of interior points in a sublattice. Can. J. Math. 43, 1022-1035 (1991) 